Pacific

Journal of

Mathematics

DUAL SPACES AND ISOMORPHISMS OF SOME DIFFERENTIAL BANACH $*$-ALGEBRAS OF OPERATORS

Edward Kissin and Victor S. Shulman 


\title{
DUAL SPACES AND ISOMORPHISMS OF SOME DIFFERENTIAL BANACH *-ALGEBRAS OF OPERATORS
}

\author{
Edward Kissin and Victor S. Shulman
}

\begin{abstract}
The paper continues the study of differential Banach *algebras $\mathcal{A}_{S}$ and $\mathcal{F}_{S}$ of operators associated with symmetric operators $S$ on Hilbert spaces $H$. The algebra $\mathcal{A}_{S}$ is the domain of the largest *-derivation $\delta_{S}$ of $B(H)$ implemented by $S$ and the algebra $\mathcal{F}_{S}$ is the closure of the set of all finite rank operators in $\mathcal{A}_{S}$ with respect to the norm $\|A\|=\|A\|+\left\|\delta_{S}(A)\right\|$. When $S$ is selfadjoint, $\mathcal{F}_{S}$ is the domain of the largest $*_{-}$ derivation of the algebra $C(H)$ implemented by $S$. If $S$ is bounded, $\mathcal{F}_{S}=C(H)$ and $\mathcal{A}_{S}=B(H)$, so $\mathcal{A}_{S}$ is isometrically isomorphic to the second dual of $\mathcal{F}_{S}$. For unbounded selfadjoint operators $S$ the paper establishes the full analogy with the bounded case: $\mathcal{A}_{S}$ is isometrically isomorphic to the second dual of $\mathcal{F}_{S}$. The paper also classifies the algebras $\mathcal{A}_{S}$ and $\mathcal{F}_{S}$ up to isometrical *-isomorphism and obtains some partial results about bounded but not necessarily isometrical *-isomorphisms of the algebras $\mathcal{F}_{S}$.
\end{abstract}

\section{Introduction and preliminaries.}

Extensive development of non-commutative geometry requires elaborating of the theory of differential Banach *-algebras, that is, dense *-subalgebras of $C^{*}$-algebras whose properties in many respects are analogous to the properties of algebras of differentiable functions.

Blackadar and Cuntz [2] and the authors [12] introduced and studied various classes of differential Banach *-algebras; the most interesting class consists of $\mathbf{D}$-algebras, that is, dense ${ }^{*}$-subalgebras $\mathcal{A}$ of $C^{*}$-algebras $(\mathfrak{U},\|\cdot\|)$ which, in turn, are Banach *-algebras with respect to another norm $\|\cdot\|_{1}$ and the norms $\|\cdot\|$ and $\|\cdot\|_{1}$ on $\mathcal{A}$ satisfy the inequality:

$$
\|x y\| \leq D\left(\|x\|\|y\|_{1}+\|x\|_{1}\|y\|\right), \quad \text { for } x, y \in \mathcal{A},
$$

for some $D>0$. This class contains, for example, the domains $D(\delta)$ of closed unbounded *-derivations $\delta$ of $C^{*}$-algebras $\mathfrak{U}$ where the norm $\|\cdot\|_{1}$ on $D(\delta)$ is defined, as usual, by the formula

$$
\|A\|_{1}=\|A\|+\|\delta(A)\|, \quad \text { for } A \in D(\delta) .
$$


Much work has been done on the investigation of properties of the differential Banach *-algebras (see Blackadar and Cuntz [2] and Kissin and Shulman $[\mathbf{1 2}, \mathbf{1 3}]$ ) and the algebras $D(\delta)$ in particular (see, for example, Bratteli and Robinson [3] and Sakai [16]).

In many cases closed ${ }^{*}$-derivations of $C^{*}$-algebras $\mathfrak{U}$ of operators on Hilbert spaces are implemented by closed symmetric operators. In particular, Bratteli and Robinson [3] showed that if $\mathfrak{U}$ contains the ideal of all compact operators then any closed *-derivation of $\mathfrak{U}$ is implemented by a symmetric operator.

Any closed symmetric operator $S$ on a Hilbert space $H$ implements closed *-derivations of various $C^{*}$-algebras of operators on $H$. Among all these derivations there is the largest one - $\delta_{S}$ with domain $D\left(\delta_{S}\right)$ (which we denote by $\mathcal{A}_{S}$ ) containing the domains of all derivations implemented by $S$ :

$$
\begin{aligned}
\mathcal{A}_{S}= & \left\{A \in B(H): A D(S) \subseteq D(S), A^{*} D(S) \subseteq D(S)\right. \text { and } \\
& \left.\left.(S A-A S)\right|_{D(S)} \text { extends to a bounded operator } A_{S}\right\} \\
& \text { and } \delta_{S}(A)=i \text { Closure }(S A-A S), \quad \text { for } A \in \mathcal{A}_{S} .
\end{aligned}
$$

The closure of $\mathcal{A}_{S}$ with respect to the norm $\|\cdot\|$ in $B(H)$ is the enveloping $C^{*}$-algebra which we denote by $\mathfrak{U}_{S}$.

The algebra $\mathcal{A}_{S}$ is a unital Banach *-algebra with respect to the norm

$$
\|A\|_{S}=\|A\|+\left\|A_{S}\right\| .
$$

If $S$ implements a ${ }^{*}$-derivation $\delta$ of a $C^{*}$-algebra $\mathfrak{U}$ of operators on $H$ then

$$
D(\delta) \subseteq \mathcal{A}_{S}, \quad \mathfrak{U} \subseteq \mathfrak{U}_{S} \text { and } \delta=\delta_{S} \mid \mathfrak{U}
$$

By $C(H)$ we denote the algebra of all compact operators on $H$. The *-algebras

$$
\mathcal{K}_{S}=\mathcal{A}_{S} \cap C(H) \quad \text { and } \quad \mathcal{J}_{S}=\left\{A \in \mathcal{K}_{S}: \delta_{S}(A) \in C(H)\right\}
$$

are dense in $C(H)$ and are the domains of the largest closed *-derivations from $C(H)$ into $B(H)$ and $C(H)$, respectively, implemented by $S$.

By $\mathcal{F}_{S}$ we denote the closure with respect to the norm $\|\cdot\|_{S}$ of the subalgebra of all finite rank operators in $\mathcal{A}_{S}$.

It was shown in $[\mathbf{1 3}]$ that $\left(\mathcal{K}_{S},\|\cdot\|_{S}\right)$ and $\left(\mathcal{J}_{S},\|\cdot\|_{S}\right)$ are semisimple Banach *-algebras, that $\left(\mathcal{F}_{S},\|\cdot\|_{S}\right)$ is a simple Banach $*_{\text {-algebra and }}$

$$
\mathcal{F}_{S} \subseteq \mathcal{J}_{S} \subseteq \mathcal{K}_{S} \subseteq \mathcal{A}_{S}
$$

Furthermore, $\mathcal{F}_{S}, \mathcal{J}_{S}$ and $\mathcal{K}_{S}$ are closed two-sided ideals of $\left(\mathcal{A}_{S},\|\cdot\|_{S}\right)$ and $\mathcal{F}_{S}$ is contained in any closed two-sided ideal of $\left(\mathcal{A}_{S},\|\cdot\|_{S}\right)$. The relation between the ideals $\mathcal{F}_{S}, \mathcal{J}_{S}$ and $\mathcal{K}_{S}$ and the question of how the properties of the operator $S$ are reflected in the structure of $\mathcal{K}_{S}, \mathcal{J}_{S}$ and $\mathcal{F}_{S}$ were investigated in [13]. In particular, it was established that $\overline{\left(\mathcal{K}_{S}\right)^{2}}=\overline{\left(\mathcal{J}_{S}\right)^{2}}=\mathcal{F}_{S}$, for all 
symmetric $S$, and that the ideals $\mathcal{J}_{S}$ and $\mathcal{F}_{S}$ have a bounded approximate identity if and only if $S$ is selfadjoint. For selfadjoint $S$, it was also proved that $\mathcal{K}_{S} \neq \mathcal{J}_{S}=\mathcal{F}_{S}$.

In spite of the fact that the structure of the algebras $\mathcal{F}_{S}, \mathcal{J}_{S}, \mathcal{K}_{S}, \mathcal{A}_{S}$ and $\mathfrak{U}_{S}$ is comparatively simple, many important questions still remain open. In Section 2 we mainly study the structure of the algebras $\mathcal{A}_{S}$ and $\mathfrak{U}_{S}$ in the case when $S$ is a selfadjoint operator. However, we also consider the case when $S$ is a symmetric operator with at least one finite deficiency index and show that the algebras $\mathcal{A}_{S}$ and $\mathfrak{U}_{S}$ contain closed ideals of finite codimension.

If $S$ is a bounded symmetric operator on $H$ then $\mathcal{F}_{S}=C(H)$ and $\mathcal{A}_{S}=$ $B(H)$, so $\mathcal{A}_{S}$ is isometrically isomorphic to the second dual of $\mathcal{F}_{S}$. In Section 3 we investigate the structure of the dual and the second dual spaces of the algebras $\mathcal{F}_{S}$ for unbounded symmetric operators $S$. In the case when $S$ is selfadjoint we establish the full analogy with the bounded case: The algebra $\mathcal{A}_{S}$ is isometrically isomorphic to the second dual of $\mathcal{F}_{S}$.

In Section 4 we study the problem of classification of the algebras $\mathcal{F}_{S}$ and $\mathcal{A}_{S}$ up to ${ }^{*}$-isomorphism. For isometrical ${ }^{*}$-isomorphism this problem is completely solved in Theorem 4.4. For bounded but not necessarily isometrical *-isomorphism we obtain some interesting partial results in the case when $S$ is selfadjoint.

\section{Structure of the algebras $\mathcal{A}_{S}$ and the enveloping $C^{*}$-algebras} $\mathfrak{U}_{S}$.

The main purpose of this section is to study the structure of the algebras $\mathcal{A}_{S}$ and $\mathfrak{U}_{S}$ in the case when $S$ is a selfadjoint operator. However, we start the section by considering the case when $S$ is a symmetric operator with at least one finite deficiency index. Making use of the existence of a $J$-symmetric representation of $\mathcal{A}_{S}$ on the deficiency space of $S$, we will show that the algebras $\mathcal{A}_{S}$ and $\mathfrak{U}_{S}$ contain closed ideals of finite codimension.

Let $S$ be symmetric, $S^{*}$ be the adjoint operator, let $N_{-}(S)$ and $N_{+}(S)$ be the deficiency spaces of $S$ and

$$
n_{ \pm}(S)=\operatorname{dim}\left(N_{ \pm}(S)\right)
$$

be the deficiency indices of $S$. It is well known that $D\left(S^{*}\right)$ is a Hilbert space with respect to the scalar product

$$
\langle x, y\rangle=(x, y)+\left(S^{*} x, S^{*} y\right), \text { for } x, y \in D\left(S^{*}\right),
$$

and it is the orthogonal sum of the closed subspaces $D(S), N_{-}(S)$ and $N_{+}(S)$ :

$$
D\left(S^{*}\right)=D(S)_{\langle+\rangle} N_{-}(S)_{\langle+\rangle} N_{+}(S)
$$


Set $N(S)=N_{-}(S)_{\langle+\rangle} N_{+}(S)$ and let $Q$ be the projection on $N(S)$ in $D\left(S^{*}\right)$. It was shown in [7] and [8] that

$$
[x, y]=i\left(x, S^{*} y\right)-i\left(S^{*} x, y\right), \text { for } x, y \in N(S),
$$

is an indefinite non-degenerate sesquilinear form on $N(S)$, that

$$
\pi_{S}(A)=\left.Q A\right|_{N(S)}, \quad \text { for } A \in \mathcal{A}_{S},
$$

is a bounded representation of $\left(\mathcal{A}_{S},\|\cdot\|_{S}\right)$ on $N(S)$ and that it is $J$-symmetric:

$$
\left[\pi_{S}(A) x, y\right]=\left[x, \pi_{S}\left(A^{*}\right) y\right], \quad \text { for } x, y \in N(S) .
$$

A subspace $L$ in $N(S)$ is neutral if

$$
[x, y]=0, \quad \text { for all } x, y \in L .
$$

The operator $S$ is well-behaved if the representation $\pi_{S}$ has no neutral invariant subspace.

Let $\kappa_{S}=\min \left(n_{-}(S), n_{+}(S)\right)$ and assume that $0<\kappa_{S}<\infty$. It was proved in [10] that the representation $\pi_{S}$ has a $\kappa_{S}$-dimensional subrepresentation $\sigma$. Let $\rho$ be an irreducible subrepresentation of $\sigma$. It was shown in [11] that $\rho$ is bounded with respect to the operator norm $\|\cdot\|$ in $\mathcal{A}_{S}$ and, therefore, extends to a bounded ${ }^{*}$-representation of the enveloping $C^{*}$-algebra $\mathfrak{U}_{S}$. If $S$ is well-behaved, it follows from Theorem 28.13 [14] that $\mathcal{K}_{S} \subseteq \operatorname{Ker}(\rho)$. This yields

Theorem 2.1. Let $S$ be a symmetric unbounded operator and $0<\kappa_{S}<\infty$.

(i) There exists a closed two-sided ideal $J$ in the Banach ${ }^{*}$-algebra $\left(\mathcal{A}_{S}\right.$, $\|\cdot\|)$ such that the quotient algebra $\mathcal{A}_{S} / J$ is isomorphic to the full matrix algebra $M_{n}(\mathbb{C})$ with $0<n \leq \kappa_{S}$.

(ii) The uniform closure $\bar{J}$ of $J$ in $\mathfrak{U}_{S}$ is a closed two-sided ideal and the quotient algebra $\mathfrak{U}_{S} / \bar{J}$ is isomorphic to the full matrix algebra $M_{n}(\mathbb{C})$. (iii) If $S$ is well-behaved then $\mathcal{K}_{S} \subseteq J$ and $C(H) \subseteq \bar{J}$.

Example 2.2. Let $H=L^{2}(0,1)$ and $S=i \frac{d}{d t}$ with domain $D(S)$ consisting of all absolutely continuous functions $h$ such that $h^{\prime} \in L^{2}(0,1)$ and $h(0)=$ $h(1)=0$. Then $S$ is a symmetric operator and $n_{-}(S)=n_{+}(S)=1$.

It was proved in [9] that $S$ is well-behaved. Therefore it follows from Theorem 2.1 that there exists a closed two-sided ideal $J$ in $\left(\mathcal{A}_{S},\|\cdot\|\right)$ containing $\mathcal{K}_{S}$ such that $\operatorname{dim}\left(\mathcal{A}_{S} / J\right)=1$ and that the uniform closure of $J$ in $\mathfrak{U}_{S}$ is an ideal of codimension 1 .

Let $S$ be the same as in Example 2.2 and let Lip $(0,1)$ be the algebra of all functions on $[0,1]$ satisfying a Lipshitz condition: $|g(t)-g(s)| \leq K_{g}|t-s|$ for some $K_{g}>0$ and all $t, s \in[0,1]$. For $g \in \operatorname{Lip}(0,1)$, denote by $M_{g}$ the operator of multiplication by $g$ on $L^{2}(0,1)$ and set $\mathcal{B}=\left\{M_{g}: g \in\right.$ $\operatorname{Lip}(0,1)\}$. Then $M_{g} D(S) \subseteq D(S),\left(M_{g}\right)^{*} D(S)=M_{\bar{g}} D(S) \subseteq D(S)$ and 
$S M_{g}-M_{g} S$ extends to the operator $i M_{g^{\prime}}$ which is bounded, since $g^{\prime}$ is essentially bounded on $[0,1]$. Thus $\mathcal{B} \subset \mathcal{A}_{S}$.

(The authors are grateful to the referee of the paper for pointing out an error in the definition of the algebra $\mathcal{B}$ in the first version of the paper.)

Problem 2.3. Is $\mathcal{A}_{S}=\mathcal{B}+\mathcal{K}_{S}$ ?

The assumption that a symmetric operator $S$ is selfadjoint makes the task of studying the structure of the algebras $\mathcal{A}_{S}$ and $\mathfrak{U}_{S}$ easier. First of all, the structure of the ideals $\mathcal{K}_{S}, \mathcal{J}_{S}$ and $\mathcal{F}_{S}$ is simpler. While for arbitrary symmetric operators $S$ it is only known (see [13]) that $\overline{\left(\mathcal{K}_{S}\right)^{2}}=$ $\overline{\left(\mathcal{J}_{S}\right)^{2}}=\mathcal{F}_{S}$, where the closure is taken with respect to the norm $\|\cdot\|_{S}$, for selfadjoint operators $S$ it was shown in [13] that $\mathcal{F}_{S}=\mathcal{J}_{S} \neq \mathcal{K}_{S}$. Secondly, in the selfadjoint case we can employ the Spectral Theorem to establish the structure of $\mathcal{A}_{S}$ and $\mathfrak{U}_{S}$.

Let

$$
S=\int_{-\infty}^{\infty} \lambda d E_{S}(\lambda)
$$

be the spectral decomposition of $S$. For every integer $n$, set

$$
P_{S}(n)=E_{S}(n+1)-E_{S}(n) \text { and }[S]=\sum_{-\infty}^{\infty} n P_{S}(n) .
$$

Then $[S]$ is a selfadjoint operator, $\operatorname{Sp}([S]) \subseteq \mathbb{Z}$ and the operator $S-[S]$ is bounded. Therefore it follows that

$$
\mathcal{A}_{S}=\mathcal{A}_{[S]}, \quad \mathcal{K}_{S}=\mathcal{K}_{[S]} \quad \text { and } \mathcal{F}_{S}=\mathcal{F}_{[S]}
$$

and the norms $\|\cdot\|_{S}$ and $\|\cdot\|_{[S]}$ are equivalent on $\mathcal{A}_{S}$. This reduces the problem of the description of the structure of the algebras $\mathcal{A}_{S}$ and $\mathfrak{U}_{S}$ to the case when $\operatorname{Sp}(S) \subseteq \mathbb{Z}$.

We denote by $\mathcal{S}_{\mathbb{Z}}$ the set of all selfadjoint operators $S$ on $H$ such that $\operatorname{Sp}(S) \subseteq \mathbb{Z}$ and set

$$
H_{S}(n)=P_{S}(n) H, \quad \text { for } n \in \operatorname{Sp}(S) .
$$

Then

$$
H=\sum_{n \in \operatorname{Sp}(S)} \oplus H_{S}(n) .
$$

We omit the proof of the following simple result.

Proposition 2.4. Let $S, T \in \mathcal{S}_{\mathbb{Z}}$. If there exists a one-to-one mapping $\varphi$ from $\operatorname{Sp}(T)$ onto $\operatorname{Sp}(S)$ such that $\operatorname{dim}\left(H_{T}(n)\right)=\operatorname{dim}\left(H_{S}(\varphi(n))\right.$, for $n \in \operatorname{Sp}(T)$, and

$$
\sup _{n \in \operatorname{Sp}(T)}|\varphi(n)-n|<\infty
$$

then there exists a unitary operator $U$ such that $\mathcal{A}_{T}=U \mathcal{A}_{S} U^{*}$. 
Let $S \in \mathcal{S}_{\mathbb{Z}}$. Every operator $A$ in $B(H)$ has a block-matrix form $A=\left(A_{i j}\right)$, $i, j \in \operatorname{Sp}(S)$, with respect to decomposition (2.3). We denote by $\mathcal{D}_{S}$ the $C^{*}$ algebra of all block-diagonal operators $A=\left(A_{i j}\right)$ in $B(H)$, that is, $A_{i j}=0$ if $i \neq j$. By $\mathcal{R}$ we denote the subalgebra of all operators $A=\left(A_{i j}\right)$ in $B(H)$ with only finite number of non-zero entries $A_{i j}$. Then, clearly,

$$
\mathcal{D}_{S} \subseteq \mathcal{A}_{S} \quad \text { and } \quad \mathcal{R}_{S} \subseteq \mathcal{A}_{S} .
$$

Let $\overline{\mathcal{R}}_{S}$ be the closure of $\mathcal{R}_{S}$ in $\left(\mathcal{A}_{S},\|\cdot\|_{S}\right)$ and let $C_{S}(H)$ be the uniform closure of $\mathcal{R}_{S}$ in $B(H)$.

Lemma 2.5. $\mathcal{D}_{S}+C_{S}(H)$ is a $C^{*}$-subalgebra of $\mathfrak{U}_{S}$ and $\mathcal{D}_{S}+\overline{\mathcal{R}}_{S}$ is a closed ${ }^{*}$-subalgebra of $\left(\mathcal{A}_{S},\|\cdot\|_{S}\right)$.

Proof. Let $\mathcal{L}$ be the uniform closure of $\mathcal{D}_{S}+\mathcal{R}_{S}$ in $B(H)$. Then $\mathcal{L}$ is a $C^{*}$-subalgebra of $\mathfrak{U}_{S}$. Since $\mathcal{R}_{S}$ is a two-sided ideal of the algebra $\mathcal{D}_{S}+\mathcal{R}_{S}$, the $C^{*}$-algebra $C_{S}(H)$ is a two-sided ideal of $\mathcal{L}$. Therefore it follows from Corollary 1.8.4 [4] that $\mathcal{D}_{S}+C_{S}(H)$ is a $C^{*}$-algebra, so $\mathcal{L}=\mathcal{D}_{S}+C_{S}(H)$.

For $A \in B(H)$, set

$$
\phi(A)=\sum_{n \in \operatorname{Sp}(S)} P_{S}(n) A P_{S}(n) \quad \text { and } \quad \widetilde{A}=A-\phi(A) .
$$

Then $\phi$ is a conditional expectation from $B(H)$ onto $\mathcal{D}_{S}$ and

$$
\|\phi(A)\| \leq\|A\| \quad \text { and } \quad\|\widetilde{A}\| \leq 2\|A\| .
$$

If $A \in \mathcal{A}_{S}$ then $\widetilde{A} \in \mathcal{A}_{S}$ and Closure $(S A-A S)=\operatorname{Closure}(S \widetilde{A}-\widetilde{A} S)$.

Assume that $\left\{A_{n}\right\}$ converge to $A$ in $\mathcal{A}_{S}$ with respect to $\|\cdot\|_{S}$. Then $\left\|A-A_{n}\right\| \rightarrow 0$ and $\|$ Closure $\left(S\left(A-A_{n}\right)-\left(A-A_{n}\right) S\right) \| \rightarrow 0$, as $n \rightarrow \infty$, and therefore, by (1.2) and (2.4),

$$
\begin{aligned}
&\left\|\widetilde{A}-\widetilde{A}_{n}\right\|_{S}=\left\|\widetilde{A}-\widetilde{A}_{n}\right\|+\left\|\operatorname{Closure}\left(S\left(\widetilde{A}-\widetilde{A}_{n}\right)-\left(\widetilde{A}-\widetilde{A}_{n}\right) S\right)\right\| \\
& \leq 2\left\|A-A_{n}\right\|+\left\|\operatorname{Closure}\left(S\left(A-A_{n}\right)-\left(A-A_{n}\right) S\right)\right\| \rightarrow 0, \\
& \text { as } n \rightarrow \infty .
\end{aligned}
$$

Hence $\widetilde{A}_{n}$ converge to $\widetilde{A}$ with respect to $\|\cdot\|_{S}$.

Suppose now that $B \in \overline{\mathcal{R}}_{S}$. Then there are $\left\{B_{n}\right\}$ in $\mathcal{R}_{S}$ converging to $B$ with respect to $\|\cdot\|_{S}$. It follows from $(2.5)$ that $\widetilde{B}_{n}$ converge to $\widetilde{B}$ with respect to $\|\cdot\|_{S}$ and, since $\widetilde{B}_{n}$ belong to $\mathcal{R}_{S}$, we obtain that $\widetilde{B} \in \overline{\mathcal{R}}_{S}$.

Finally, let $C_{n}=A_{n}+B_{n}$ converge to $C$ in $\mathcal{A}_{S}$ with respect to $\|\cdot\|_{S}$ where $A_{n} \in \mathcal{D}_{S}$ and $B_{n} \in \overline{\mathcal{R}}_{S}$. Then $\widetilde{C}_{n}=\widetilde{B}_{n}$ and, by (2.5), $\widetilde{B}_{n}$ converge to $\widetilde{C}$ with respect to $\|\cdot\|_{S}$. Since, by the above argument, all $\widetilde{B}_{n}$ belong to $\overline{\mathcal{R}}_{S}$, the operator $\widetilde{C}$ also belong to $\overline{\mathcal{R}}_{S}$. Hence $C \in \mathcal{D}_{S}+\overline{\mathcal{R}}_{S}$ and $\mathcal{D}_{S}+\overline{\mathcal{R}}_{S}$ is a closed ${ }^{*}$-subalgebra of $\left(\mathcal{A}_{S},\|\cdot\|_{S}\right)$. 
Let $S \in \mathcal{S}_{\mathbb{Z}}$. We number the elements of $\operatorname{Sp}(S)$ in such a way that $\operatorname{Sp}(S)=$ $\left\{n_{i}\right\}_{i \in I}$ is an increasing sequence,

$$
0 \leq n_{i}, \text { for } 0 \leq i, \text { and } 0>n_{i} \text {, for } 0>i .
$$

Then $|i| \leq\left|n_{i}\right|$ and, depending on $S$, the set $I$ is either the set $\mathbb{Z}$ of all integers, or the set of all integers from $-\infty$ to some $m$, or from $m$ to $\infty$. We consider the case when $I=\mathbb{Z}$. Two other cases can be considered similarly.

Set

$$
\rho_{S}(k)=\left(\inf _{i \in \mathbb{Z}}\left|n_{i+k}-n_{i}\right|\right)^{-1}, \quad \text { for } k \neq 0, \quad \text { and } \rho_{S}(0)=0 .
$$

Since $\inf _{i \in \mathbb{Z}}\left|n_{i+k}-n_{i}\right| \geq|k|$,

$$
0<\rho_{S}(k) \leq \frac{1}{|k|}, \quad \text { for } k \neq 0 .
$$

Proposition 2.6. If

$$
\begin{gathered}
\lim _{|i| \rightarrow \infty}\left(n_{i+1}-n_{i}\right)=\infty \\
\text { and } \sum_{k \in \mathbb{Z}} \rho(k) \text { converges }
\end{gathered}
$$

then $\mathfrak{U}_{S}=\mathcal{D}_{S}+C_{S}(H)$.

Proof. Let $A=\left(A_{i j}\right) \in \mathcal{A}_{S}$, where $A_{i j}$ are bounded operators from $H_{S}\left(n_{j}\right)$ into $H_{S}\left(n_{i}\right)$. Then the operator

$$
B=S A-A S=\left(B_{i j}\right), \quad \text { where } B_{i j}=\left(n_{i}-n_{j}\right) A_{i j},
$$

is bounded. Set $b=\|B\|$. Since $\left\|B_{i j}\right\| \leq\|B\|$, for all $i, j \in \mathbb{Z}$,

$$
\left\|A_{i j}\right\| \leq \frac{b}{\left|n_{i}-n_{j}\right|}, \quad \text { for } i \neq j .
$$

For $k \in \mathbb{Z} \backslash 0$ and $m>0$, let

$$
G_{i j}^{k m}=A_{i j} \text {, if } j=i+k \text { and }-m \leq i \leq m \text {, and } G_{i j}^{k m}=0 \text { otherwise. }
$$

Then the operator $G^{k m}=\left(G_{i j}^{k m}\right)$ belongs to $\mathcal{R}_{S}$. Taking into account (2.6) and (2.8), we obtain that the operators $G^{k m}$ converge uniformly in $B(H)$ to a bounded operator $G^{k}=\left(G_{i j}^{k}\right)$, as $m \rightarrow \infty$, where

$$
G_{i j}^{k}=A_{i j}, \text { if } j=i+k \text {, and } G_{i j}^{k}=0 \text { otherwise. }
$$

Therefore $G^{k} \in C_{S}(H)$ and, by (2.8),

$$
\left\|G^{k}\right\|=\sup _{i}\left\|A_{i i+k}\right\| \leq b \rho_{S}(k) .
$$

It follows from (2.7) that the operator $G=\sum_{k \in \mathbb{Z} \backslash 0} G^{k}$ belongs to $C_{S}(H)$. Since $A-G \in \mathcal{D}_{S}$, we obtain that $A \in \mathcal{D}_{S}+C_{S}(H)$, so that $\mathcal{A}_{S} \subseteq \mathcal{D}_{S}+C_{S}(H)$. It follows from Lemma 2.5 that $\mathfrak{U}_{S}=\mathcal{D}_{S}+C_{S}(H)$. 
Corollary 2.7. If there are $a>0, c>0$ and an integer $N$ such that

$$
c|i|^{a} \leq n_{i+1}-n_{i} \quad \text { for } N \leq|i|
$$

then $\mathfrak{U}_{S}=\mathcal{D}_{S}+C_{S}(H)$.

Proof. Condition (2.6), clearly, holds. Let $k>4 N$. Then

$$
\begin{aligned}
\rho_{S}(k)^{-1} & =\inf _{i \in \mathbb{Z}}\left|n_{i+k}-n_{i}\right|=\inf _{i \in \mathbb{Z}} \sum_{p=1}^{k}\left(n_{i+p}-n_{i+p-1}\right) \\
& \geq c \sum_{m=N}^{\left[\frac{k}{2}\right]} m^{a} \geq \frac{c}{a+1}\left(\left[\frac{k}{2}\right]^{a+1}-(N-1)^{a+1}\right) \\
& \geq \frac{c}{a+1}\left(\frac{k}{4}\right)^{a+1} .
\end{aligned}
$$

Similarly, if $k<-2 N$ then $\rho_{S}(k)^{-1} \geq \frac{c}{a+1}\left(\frac{|k|}{4}\right)^{a+1}$. Therefore condition (2.7) also holds and the result follows from Proposition 2.6.

Suppose now that $\operatorname{dim}\left(H_{S}(n)\right)=\infty$ for all $n \in \operatorname{Sp}(S)$ and let $n_{0} \in \operatorname{Sp}(S)$. Set $K=H_{S}\left(n_{0}\right)$. Then there exists a Hilbert space $\mathcal{H}$ with $\operatorname{dim}(\mathcal{H})=\infty$ such that the $C^{*}$-algebra $C_{S}(H)$ is isomorphic to the tensor product $B(K) \otimes C(\mathcal{H})$ where $C(\mathcal{H})$ is the $C^{*}$-algebra of all compact operators on $\mathcal{H}$. Choosing a basis $\left\{e_{n}\right\}_{n=1}^{\infty}$ in $\mathcal{H}$, we obtain that the algebra $\mathcal{D}_{S}$ is isomorphic to the von Neumann algebra tensor product $B(K) \bar{\otimes} \mathcal{L}$ of $B(K)$ and the $W^{*}$-algebra $\mathcal{L}$ of all operators on $\mathcal{H}$ diagonal with respect to $\left\{e_{n}\right\}_{n=1}^{\infty}$. From this and from Proposition 2.6 we obtain the following result.

Corollary 2.8. Let $S \in \mathcal{S}_{\mathbb{Z}}$. If $\operatorname{dim}\left(H_{S}(n)\right)=\infty$ for all $n \in \operatorname{Sp}(S)$ and conditions (2.6) and (2.7) hold then there exist Hilbert spaces $K$ and $\mathcal{H}$ such that $\mathfrak{U}_{S}$ is isomorphic to $B(K) \bar{\otimes} \mathcal{L}+B(K) \otimes C(\mathcal{H})$, where $\mathcal{L}$ is the $W^{*}$-algebra of all operators on $\mathcal{H}$ diagonal with respect to some basis.

Assume now that $\operatorname{dim}\left(H_{S}(n)\right)<\infty$ for all $n \in \operatorname{Sp}(S)$. Then $C_{S}(H)$ coincides with the algebra $C(H)$ of all compact operators on $H$. Taking into account the definition of the ideal $\mathcal{K}_{S}$ and applying Proposition 2.6 we obtain the following result.

Corollary 2.9. Let $S \in \mathcal{S}_{\mathbb{Z}}$ and $\operatorname{dim}\left(H_{S}(n)\right)<\infty$ for all $n \in \operatorname{Sp}(S)$. If conditions (2.6) and (2.7) hold then $\mathfrak{U}_{S}=\mathcal{D}_{S}+C(H)$ and $\mathcal{A}_{S}=\mathcal{D}_{S}+\mathcal{K}_{S}$.

Example 2.10. Let $\left\{e_{i}\right\}_{i=-\infty}^{\infty}$ be an orthonormal basis in $H$ and let

$$
S e_{i}=\operatorname{sgn}(i)|i|^{1+a} e_{i}, \text { where } a>0 .
$$

Then $S \in \mathcal{S}_{\mathbb{Z}}$ and $n_{i}=\operatorname{sgn}(i)|i|^{1+a}$, so that

$$
\lim _{|i| \rightarrow \infty} \frac{n_{i+1}-n_{i}}{\operatorname{sgn}(i)|i|^{a}}=1+a .
$$


Therefore, by Corollaries 2.7 and 2.9, $\mathfrak{U}_{S}=\mathcal{D}_{S}+C(H)$ and $\mathcal{A}_{S}=\mathcal{D}_{S}+\mathcal{K}_{S}$ where $\mathcal{D}_{S}$ is the algebra of all operators diagonal with respect to $\left\{e_{i}\right\}_{i=-\infty}^{\infty}$. Thus the quotient algebra $\mathcal{A}_{S} / \mathcal{K}_{S}$ is isomorphic to the commutative $C^{*}$-algebra $\mathcal{D}_{S} / \mathfrak{L}$ where $\mathfrak{L}$ is the algebra of all compact diagonal operators on $H$.

Let $\left\{e_{i}\right\}_{i=-\infty}^{\infty}$ be an orthonormal basis in $H$ and let

$$
S e_{i}=i e_{i} \text { and } U e_{i}=e_{i+1}, \quad \text { for all } i \in \mathbb{Z} .
$$

Then $S \in \mathcal{S}_{\mathbb{Z}}$ and $U$ is the shift operator. We have that

$$
U D(S) \subseteq D(S), U^{*} D(S) \subseteq D(S) \text { and }\left.(S U-U S)\right|_{D(S)} \text { extends to } U,
$$

so that $U \in \mathcal{A}_{S}$. Hence $\mathfrak{U}_{S}$ contains the $C^{*}$-algebra $C\left(\mathcal{D}_{S}, U\right)$ generated by $U$ and by the commutative algebra $\mathcal{D}_{S}$ of all operators diagonal with respect to $\left\{e_{i}\right\}_{i=-\infty}^{\infty}$.

Problem 2.11. Is $\mathfrak{U}_{S}=C\left(\mathcal{D}_{S}, U\right)$ ?

\section{Dual and second dual spaces of the algebras $\mathcal{F}_{S}$.}

Let $S$ be a closed symmetric operator. Recall that $\mathcal{F}_{S}$ is the closure with respect to the norm $\|\cdot\|_{S}$ (see (1.2)) of the subalgebra of all finite rank operators in $\mathcal{A}_{S}$. If $S$ is a bounded symmetric operator on $H$, it follows that $\mathcal{F}_{S}=C(H)$ and $\mathcal{A}_{S}=B(H)$, so that $\mathcal{A}_{S}$ is isometrically isomorphic to the second dual of $\mathcal{F}_{S}$. In this section we study the structure of the dual and the second dual spaces of the algebra $\mathcal{F}_{S}$ for unbounded symmetric operators $S$. In the case when $\mathrm{S}$ is selfadjoint we establish the full analogy with the bounded case: The algebra $\mathcal{A}_{S}$ is isometrically isomorphic to the second dual of $\mathcal{F}_{S}$.

By $T(H)$ we denote the Banach *-algebra of trace class operators on $H$ with the norm

$$
|A|=\sum_{i=1}^{\infty} s_{i}(A)=\operatorname{Tr}\left(\left(A^{*} A\right)^{1 / 2}\right),
$$

where $\left\{s_{i}(A)\right\}_{i=1}^{\infty}$ is the set of all eigenvalues of the positive compact operator $\left(A^{*} A\right)^{1 / 2}$.

It is well known that $T(H)$ can be identified with the dual space of the algebra $C(H)$ : For any $T \in T(H)$,

$$
F_{T}(A)=\operatorname{Tr}(A T), \quad A \in C(H),
$$

is a bounded linear functional on $C(H)$ and $\left\|F_{T}\right\|=|T|$; and that $B(H)$ can be identified with the dual space of $T(H)$ : For any $B \in B(H)$,

$$
\theta_{B}(T)=\operatorname{Tr}(B T), \quad T \in T(H),
$$

is a bounded linear functional on $T(H)$ and $\|\theta\|=\|B\|$. 
Set $\widehat{B}(H)=B(H) \oplus B(H)$ and $\widehat{C}(H)=C(H) \oplus C(H)$. Then $\widehat{B}(H)$ and $\widehat{C}(H)$ are Banach spaces with the norm

$$
\|A \oplus B\|=\|A\|+\|B\| .
$$

Set $\widehat{T}(H)=T(H) \oplus T(H)$. It is a Banach space with the norm

$$
|R \oplus T|=\max (|R|,|T|), \quad T, R \in T(H),
$$

and it can be identified with the dual space of $\widehat{C}(H)$ : For $R, T \in T(H)$,

$$
F_{R \oplus T}(A \oplus B)=\operatorname{Tr}(A R)+\operatorname{Tr}(B T), \quad A \oplus B \in \widehat{C}(H),
$$

is a bounded linear functional on $\widehat{C}(H)$ and $\left\|F_{R \oplus T}\right\|=|R \oplus T|$. Similarly, $\widehat{B}(H)$ can be identified with the dual space of $\widehat{T}(H)$ : For $A, B \in B(H)$,

$$
\theta_{A \oplus B}(R \oplus T)=\operatorname{Tr}(A R)+\operatorname{Tr}(B T), \quad R \oplus T \in \widehat{T}(H),
$$

is a bounded linear functional on $\widehat{T}(H)$ and $\left\|\theta_{A \oplus B}\right\|=\|A \oplus B\|$.

Set

$$
\widehat{\mathcal{A}}_{S}=\left\{A \oplus A_{S}: A \in \mathcal{A}_{S}\right\} \quad \text { and } \quad \widehat{\mathcal{F}}_{S}=\left\{A \oplus A_{S}: A \in \mathcal{F}_{S}\right\},
$$

where $A_{S}=$ Closure $(S A-A S)$. Then $\left(\mathcal{A}_{S},\|\cdot\|_{S}\right)$ and $\left(\widehat{\mathcal{A}}_{S},\|\cdot\|\right),\left(\mathcal{F}_{S},\|\cdot\|_{S}\right)$ and $\left(\widehat{\mathcal{F}}_{S},\|\cdot\|\right)$ are isometrically isomorphic, since

$$
\|A\|_{S}=\|A\|+\left\|A_{S}\right\|=\left\|A \oplus A_{S}\right\| .
$$

Therefore $\widehat{\mathcal{A}}_{S}$ is a closed subspace of $\widehat{B}(H)$ and $\widehat{\mathcal{F}}_{S}$ is a closed subspace of $\widehat{C}(H)$, since $A \in \mathcal{F}_{S}$ implies $A_{S} \in C(H)$.

Set

$$
\begin{array}{r}
\mathfrak{T}_{S}=\left\{T \in T(H): T D(S) \subseteq D\left(S^{*}\right), T^{*} D(S) \subseteq D\left(S^{*}\right)\right. \text { and the operator } \\
\left.\left.\left(S^{*} T-T S\right)\right|_{D(S)} \text { extends to a bounded trace class operator } \mathbb{T}\right\} .
\end{array}
$$

If $T \in \mathfrak{T}_{S} \cap \mathcal{A}_{S}$ then $\mathbb{T}_{S}=T_{S}$. In particular, if $S$ is selfadjoint then $\mathbb{T}_{S}=T_{S}$ for all $T \in \mathfrak{T}_{S}$. Clearly, $\mathfrak{T}_{S}$ is a linear subspace in $T(H)$ and

$$
\check{\mathfrak{T}}_{S}=\left\{\mathbb{T}_{S} \oplus T: T \in \mathfrak{T}_{S}\right\}
$$

is a linear subspace in $\widehat{T}(H)$. For $T \in \mathfrak{T}_{S}$ and $z, u \in D(S)$,

$$
-\left(\left(\mathbb{T}_{S}\right)^{*} z, u\right)=-\left(z, \mathbb{T}_{S} u\right)=-\left(z,\left(S^{*} T-T S\right) u\right)=\left(\left(S^{*} T^{*}-T^{*} S\right) z, u\right),
$$

so that

$$
-\left.\left(\mathbb{T}_{S}\right)^{*}\right|_{D(S)}=\left.\left(S^{*} T^{*}-T^{*} S\right)\right|_{D(S)}=\left.\left(\mathbb{T}^{*}\right)_{S}\right|_{D(S)} .
$$

Therefore $T^{*} \in \mathfrak{T}_{S}$.

For $x, y \in H$, the rank one operator $x \otimes y$ on $H$ is defined by the formula

$$
(x \otimes y) z=(z, x) y .
$$


It is easy to check that

$$
\begin{gathered}
\|x \otimes y\|=\|x\|\|y\|, \\
(x \otimes y)^{*}=y \otimes x,(x \otimes y)(u \otimes v)=(v, x)(u \otimes y), \\
R(x \otimes y)=x \otimes R y, \text { and }(x \otimes y) R \text { extends to }\left(R^{*} x\right) \otimes y,
\end{gathered}
$$

if $R$ is a densely defined operator, $y \in D(R)$ and $x \in D\left(R^{*}\right)$. Let $\left\{e_{j}\right\}_{j=1}^{\infty}$ be a basis in $H$. Then

$$
\begin{aligned}
\operatorname{Tr}(x \otimes y) & =\sum_{j=1}^{\infty}\left((x \otimes y) e_{j}, e_{j}\right)=\sum_{j=1}^{\infty}\left(e_{j}, x\right)\left(y, e_{j}\right) \\
& =\left(y, \sum_{j=1}^{\infty}\left(x, e_{j}\right) e_{j}\right)=(y, x) .
\end{aligned}
$$

Let $x, y \in D\left(S^{*}\right)$ and $T=x \otimes y$. By (3.4) and (3.5),

$$
\begin{gathered}
T z=(z, x) y \in D\left(S^{*}\right) \\
T^{*} z=(y \otimes x) z=(z, y) x \in D\left(S^{*}\right), \text { for } z \in H, \\
\text { and } \mathbb{T}_{S}=S^{*} T-T S=x \otimes S^{*} y-\left(S^{*} x\right) \otimes y \in T(H),
\end{gathered}
$$

so that $T \in \mathfrak{T}_{S}$. By $\Phi_{S}$ we denote the set of all linear combinations of the operators $x \otimes y$, for $x, y \in D\left(S^{*}\right)$. Clearly, $\Phi \subset \mathfrak{T}_{S}$ and

$$
\check{\Phi}_{S}=\left\{\mathbb{T}_{S} \oplus T: T \in \Phi_{S}\right\}
$$

is a linear subspace of $\check{\mathfrak{T}}_{S}$.

Let $X^{*}$ be the dual space of a Banach space $X$ and $Y$ be a linear subspace of $X$. The annihilator

$$
Y^{\perp}=\left\{F \in X^{*}: F(y)=0, \text { for all } y \in Y\right\}
$$

of $Y$ in $X^{*}$ is a closed subspace of $X^{*}$ and from the general theory of Banach spaces (see [5] II.4.18 and [15] III, Problem 30) we have the following lemma.

Lemma 3.1. The dual space $Y^{*}$ of a closed subspace $Y$ of $X$ is isometrically isomorphic to the quotient space $X^{*} / Y^{\perp}$ and the second dual $Y^{* *}$ of $Y$ is isometrically isomorphic to $Y^{\perp \perp}$ where

$$
Y^{\perp \perp}=\left\{\theta \in X^{* *}: \theta(F)=0, \text { for all } F \in Y^{\perp}\right\} .
$$

Since $\widehat{\mathcal{F}}_{S} \subseteq \widehat{C}(H)$, the annihilator $\left(\widehat{\mathcal{F}}_{S}\right)^{\perp}$ is a closed subspace of the dual space $\widehat{C}(H)^{*}=\widehat{T}(H)$ and, since $\check{\Phi}_{S} \subseteq \check{\mathfrak{T}}_{S} \subseteq \widehat{T}(H)$, the annihilator $\left(\check{\Phi}_{S}\right)^{\perp}$ is a closed subspace of the dual space $\widehat{T}(H)^{*}=\widehat{B}(H)$.

Theorem 3.2. (i) $\check{\mathfrak{T}}_{S}$ is a closed subspace in $\widehat{T}(H)$ and $\left(\widehat{\mathcal{F}}_{S}\right)^{\perp}=\check{\mathfrak{T}}_{S}$. (ii) $\left(\check{\mathfrak{T}}_{S}\right)^{\perp} \subseteq\left(\check{\Phi}_{S}\right)^{\perp}=\left\{A \oplus A_{S}: A \in \mathcal{A}_{S}\right.$ and $\left.A D\left(S^{*}\right) \subseteq D(S)\right\} \subseteq \widehat{\mathcal{A}}_{S}$. 
Proof. Let $\mathbb{T}_{S} \oplus T \in \check{\mathfrak{T}}_{S}$ and $x, y \in D(S)$. Then $A=x \otimes y \in \mathcal{F}_{S}$ and, by (3.3) and (3.5),

$$
\begin{aligned}
A_{S} & =S(x \otimes y)-(x \otimes y) S=x \otimes S y-(S x) \otimes y, \\
A_{S} T & =(x \otimes S y) T-((S x) \otimes y) T=\left(T^{*} x\right) \otimes S y-\left(T^{*} S x\right) \otimes y, \\
A \mathbb{T}_{S} & =(x \otimes y) \mathbb{T}_{S}=\left(\left(\mathbb{T}_{S}\right)^{*} x\right) \otimes y=\left(\left(T^{*} S-S^{*} T^{*}\right) x\right) \otimes y .
\end{aligned}
$$

Therefore, by (3.1), (3.6) and (3.8),

$$
\begin{aligned}
F_{\mathbb{T}_{S} \oplus T}\left(A \oplus A_{S}\right) & =\operatorname{Tr}\left(A \mathbb{T}_{S}\right)+\operatorname{Tr}\left(A_{S} T\right) \\
& =\left(y,\left(T^{*} S-S^{*} T^{*}\right) x\right)+\left(S y, T^{*} x\right)-\left(y, T^{*} S x\right)=0 .
\end{aligned}
$$

It follows from Lemma 3.1 [13] that any finite rank operator $A$ in $\mathcal{F}_{S}$ has the form $A=\sum_{i=1}^{n} x_{i} \otimes y_{i}$ where $x_{i}, y_{i} \in D(S)$. Hence $F_{\mathbb{T}_{S} \oplus T}(A \oplus$ $\left.A_{S}\right)=0$ for any finite rank operator $A \in \mathcal{F}_{S}$. Since, by definition of $\mathcal{F}_{S}$, finite rank operators are dense in $\left(\mathcal{F}_{S},\|\cdot\|_{S}\right)$ and since $\left(\mathcal{F}_{S},\|\cdot\|_{S}\right)$ and $\left(\widehat{\mathcal{F}}_{S},\|\cdot\|\right)$ are isometrically isomorphic, the operators $A \oplus A_{S}$, where $A$ are finite rank operators, are dense in $\widehat{\mathcal{F}}_{S}$. Since $F_{\mathbb{T}_{S} \oplus T}$ is continuous on $\widehat{C}(H)$, $F_{\mathbb{T}_{S} \oplus T}\left(A \oplus A_{S}\right)=0$, for all $A \in \mathcal{F}_{S}$. Therefore $F_{\mathbb{T}_{S} \oplus T} \in\left(\widehat{\mathcal{F}}_{S}\right)^{\perp}$, so that $\check{\mathfrak{T}}_{S} \subseteq\left(\widehat{\mathcal{F}}_{S}\right)^{\perp}$.

Conversely, let $R \oplus T \in\left(\widehat{\mathcal{F}}_{S}\right)^{\perp} \subseteq \widehat{T}(H)$ and let $A=x \otimes y \in \mathcal{F}_{S}$, where $x, y \in D(S)$. From (3.1), (3.5), (3.6) and (3.8) it follows that

$$
\begin{aligned}
0 & =F_{R \oplus T}\left(A \oplus A_{S}\right)=\operatorname{Tr}(A R)+\operatorname{Tr}\left(A_{S} T\right) \\
& =\operatorname{Tr}\left(\left(R^{*} x\right) \otimes y\right)+\operatorname{Tr}\left[\left(T^{*} x\right) \otimes S y-\left(T^{*} S x\right) \otimes y\right] \\
& =\left(y, R^{*} x\right)+\left(S y, T^{*} x\right)-\left(y, T^{*} S x\right) .
\end{aligned}
$$

Hence

$$
\left(S y, T^{*} x\right)=\left(y,\left(T^{*} S-R^{*}\right) x\right), \quad \text { for all } x, y \in D(S) .
$$

Therefore $T^{*} x \in D\left(S^{*}\right)$ and $S^{*} T^{*} x=\left(T^{*} S-R^{*}\right) x$. Thus $T^{*} D(S) \subseteq D\left(S^{*}\right)$ and

$$
(S x, T y)=\left(T^{*} S x, y\right)=\left(S^{*} T^{*} x, y\right)+\left(R^{*} x, y\right)=(x, T S y)+(x, R y) .
$$

From this it follows that $T y \in D\left(S^{*}\right)$ and $S^{*} T y=T S y+R y$. Hence

$$
T D(S) \subseteq D\left(S^{*}\right) \quad \text { and }\left.\quad R\right|_{D(S)}=\left.S^{*} T\right|_{D(S)}-\left.T S\right|_{D(S)} .
$$

Therefore $T \in \mathfrak{T}_{S}$ and $R=\mathbb{T}_{S}$. Thus $\left(\widehat{\mathcal{F}}_{S}\right)^{\perp} \subseteq \check{\mathfrak{T}}_{S}$, so that $\left(\widehat{\mathcal{F}}_{S}\right)^{\perp}=\check{\mathfrak{T}}_{S}$. From this we also obtain that $\check{\mathfrak{T}}_{S}$ is a closed subspace of $\widehat{T}(H)$. Part (i) is proved.

Since $\check{\Phi}_{S} \subseteq \check{\mathfrak{T}}_{S}$, we have $\left(\check{\mathfrak{T}}_{S}\right)^{\perp} \subseteq\left(\check{\Phi}_{S}\right)^{\perp}$. Let now $A \oplus A_{S} \in \widehat{\mathcal{A}}_{S}$ and $A D\left(S^{*}\right) \subseteq D(S)$. It was shown in Lemma $3.1[\mathbf{1 3}]$ that

$$
\left.A_{S}\right|_{D\left(S^{*}\right)}=\left.\left(S^{*} A-A S^{*}\right)\right|_{D\left(S^{*}\right)}
$$


For $x, y \in D\left(S^{*}\right)$, the operator $T=x \otimes y$ belongs to $\Phi_{S}$ and, taking the above equality into account, we obtain from (3.5) and (3.7) that

$$
\begin{aligned}
& A_{S} T=x \otimes A_{S} y=x \otimes\left(S^{*} A-A S^{*}\right) y \quad \text { and } \\
& A \mathbb{T}_{S}=A\left(x \otimes S^{*} y-\left(S^{*} x\right) \otimes y\right)=x \otimes A S^{*} y-\left(S^{*} x\right) \otimes A y .
\end{aligned}
$$

Therefore, by (3.2) and (3.6),

$$
\begin{aligned}
\theta_{A \oplus A_{S}}\left(\mathbb{T}_{S} \oplus T\right) & =\operatorname{Tr}\left(A \mathbb{T}_{S}\right)+\operatorname{Tr}\left(A_{S} T\right) \\
& =\left(A S^{*} y, x\right)-\left(A y, S^{*} x\right)+\left(S^{*} A y, x\right)-\left(A S^{*} y, x\right) \\
& =\left(S^{*} A y, x\right)-\left(A y, S^{*} x\right) .
\end{aligned}
$$

Since $A D\left(S^{*}\right) \subseteq D(S)$, it follows that $S^{*} A y=S A y$ and $\left(A y, S^{*} x\right)=$ $(S A y, x)$. Hence $\theta_{A \oplus A_{S}}\left(\mathbb{T}_{S} \oplus T\right)=0$ and, by linearity, it holds for all $T \in \Phi_{S}$. Therefore

$$
\left\{A \oplus A_{S}: A \in \mathcal{A}_{S} \text { and } A D\left(S^{*}\right) \subseteq D(S)\right\} \subseteq\left(\check{\Phi}_{S}\right)^{\perp} .
$$

Conversely, let $A \oplus B \in\left(\check{\Phi}_{S}\right)^{\perp}$. Then, for every $x, y \in D\left(S^{*}\right), T=x \otimes y \in$ $\Phi_{S}$ and

$$
\theta_{A \oplus B}\left(\mathbb{T}_{S} \oplus T\right)=\operatorname{Tr}\left(A T_{S}\right)+\operatorname{Tr}(B T)=0 .
$$

By (3.5), $B T=x \otimes B y$ and, as above, $A \mathbb{T}_{S}=x \otimes A S^{*} y-\left(S^{*} x\right) \otimes A y$. Hence, by $(3.6)$,

$$
0=\left(A S^{*} y, x\right)-\left(A y, S^{*} x\right)+(B y, x) .
$$

Thus

$$
\left(A y, S^{*} x\right)=\left(A S^{*} y, x\right)+(B y, x), \quad \text { for all } x, y \in D\left(S^{*}\right) .
$$

Therefore $A y \in D\left(S^{* *}\right)$ and $S^{* *} A y=A S^{*} y+B y$. Since $S$ is closed, $S^{* *}=S$ and we obtain that

$$
A D\left(S^{*}\right) \subseteq D(S) \quad \text { and }\left.\quad B\right|_{D\left(S^{*}\right)}=\left.\left(S A-A S^{*}\right)\right|_{D\left(S^{*}\right)} .
$$

Restricting (3.10) to $\mathrm{D}(\mathrm{S})$, we have

$$
A D(S) \subseteq D(S) \quad \text { and }\left.\quad B\right|_{D(S)}=\left.(S A-A S)\right|_{D(S)} .
$$

Making use of (3.10), we obtain that for $z \in D(S)$ and $u \in D\left(S^{*}\right)$,

$$
\left(A^{*} z, S^{*} u\right)=\left(z, A S^{*} u\right)=(z, S A u)-(z, B u)=\left(A^{*} S z, u\right)-\left(B^{*} z, u\right) .
$$

Therefore $A^{*} z \in D\left(S^{* *}\right)$. Since $S^{* *}=S$, we have $A^{*} D(S) \subseteq D(S)$. Thus $A \in \mathcal{A}_{S}$ and $B=A_{S}$, so $A \oplus B=A \oplus A_{S} \in \widehat{\mathcal{A}}_{S}$. Taking into account that $A D\left(S^{*}\right) \subseteq D(S)$, we obtain that

$$
\left(\check{\Phi}_{S}\right)^{\perp} \subseteq\left\{A \oplus A_{S}: A \in \mathcal{A}_{S} \text { and } A D\left(S^{*}\right) \subseteq D(S)\right\} .
$$

Combining this with (3.9), we complete the proof of the theorem.

Since the Banach spaces $\left(\mathcal{F}_{S},\|\cdot\|_{S}\right)$ and $\left(\widehat{\mathcal{F}}_{S},\|\cdot\|\right)$ and the Banach spaces $\left(\mathcal{A}_{S},\|\cdot\|_{S}\right)$ and $\left(\widehat{\mathcal{A}}_{S},\|\cdot\|\right)$ are isometrically isomorphic and since $\left(\widehat{\mathcal{F}}_{S},\|\cdot\|\right)$ is a closed subspace of $\widehat{C}(H)$, Lemma 3.1 and Theorem 3.2 yield: 
Corollary 3.3. The dual space of the Banach ${ }^{*}$-algebra $\left(\mathcal{F}_{S},\|\cdot\|_{S}\right)$ is isometrically isomorphic to the quotient space $\widehat{T}(H) / \check{\mathfrak{T}}_{S}$ and the second dual space of $\left(\mathcal{F}_{S},\|\cdot\|_{S}\right)$ is isometrically isomorphic to a closed subspace of $\left(\mathcal{A}_{S},\|\cdot\|_{S}\right)$.

The following example shows that if $S$ is not selfadjoint then, generally speaking, $\left(\check{\Phi}_{S}\right)^{\perp} \neq \widehat{\mathcal{A}}_{S}$, so that $\left(\mathcal{F}_{S}\right)^{\perp \perp} \neq \widehat{\mathcal{A}}_{S}$ and the second dual space of $\left(\mathcal{F}_{S},\|\cdot\|_{S}\right)$ is isometrically isomorphic to a proper subspace of $\left(\mathcal{A}_{S},\|\cdot\|_{S}\right)$.

Example 3.4. Let, as in Example 2.2, $H=L^{2}(0,1)$ and the operator $S=$ $i \frac{d}{d t}$ with domain $D(S)=\left\{h(t): h, h^{\prime} \in L_{2}(0,1)\right.$ and $\left.h(0)=h(1)=0\right\}$. Then $S$ is a symmetric operator, non-selfadjoint and

$$
D\left(S^{*}\right)=\left\{h(t): h, h^{\prime} \in L^{2}(0,1)\right\} .
$$

Let $g(t)$ be a differentiable function on $[0,1]$ such that $g(0) \neq 0$ and let $M_{g}$ be the bounded operator of multiplication by $g(t)$ on $H$. Then $M_{g} \in \mathcal{A}_{S}$. If $h(t) \in D\left(S^{*}\right)$ and $h(0) \neq 0$ then $\left(M_{g} h\right)(0)=g(0) h(0) \neq 0$, so that $M_{g} h \notin D(S)$. Thus $M_{g} \oplus\left(M_{g}\right)_{S} \notin\left\{A \oplus A_{S}: A \in \mathcal{A}_{S}\right.$ and $\left.A D\left(S^{*}\right) \subseteq D(S)\right\}$. Hence $\left(\check{\Phi}_{S}\right)^{\perp} \neq \mathcal{A}_{S}$.

Assume now that $S$ is selfadjoint. Then $D\left(S^{*}\right)=D(S), \mathbb{T}_{S}=T_{S}$, for $T \in \mathfrak{T}_{S}$, and

$$
\mathfrak{T}_{S}=\left\{T \in T(H) \cap \mathcal{A}_{S}: T_{S} \in T(H)\right\} \subseteq \mathcal{A}_{S} .
$$

It is well known (see, for example, [5] and [6]) that the algebra $T(H)$ is a two-sided ideal of $B(H)$ and if $A \in B(H)$ and $B \in T(H)$ then

$$
|A B| \leq\|A\||B|, \quad\left|B^{*}\right|=|B| \quad \text { and } \quad\|B\| \leq|B| .
$$

We consider now two equivalent norms on $\mathfrak{T}_{S}$ :

$$
|T|_{1}=|T|+\left|T_{S}\right| \text { and }|T|_{2}=\max \left(|T|,\left|T_{S}\right|\right), \text { for } T \in \mathfrak{T}_{S} .
$$

Since

$$
\mathbb{T}_{S}=T \text { and }|T|_{2}=\max \left(|T|,\left|T_{S}\right|\right)=\left|\mathbb{T}_{S} \oplus T\right|, \text { for } T \in \mathfrak{T}_{S},
$$

$\left(\mathfrak{T}_{S},|\cdot|_{2}\right)$ is isometrically isomorphic to $\check{\mathfrak{T}}_{S}$.

Proposition 3.5. Let $S$ be selfadjoint. Then:

(i) $\mathfrak{T}_{S} \subset \mathcal{F}_{S}$ and $\left(\mathfrak{T}_{S},|\cdot|_{2}\right)$ is a two-sided Banach $\mathcal{A}_{S}$-module;

(ii) $\left(\mathfrak{T}_{S},|\cdot|_{1}\right)$ is a Banach *-algebra and a $\mathbf{D}$-subalgebra of $C(H)$ (see (1.1)) with $D=1$.

Proof. It was shown in [13] that if $S$ is selfadjoint then $\mathcal{F}_{S}$ coincides with the algebra $\mathcal{J}_{S}=\left\{A \in \mathcal{A}_{S}: A\right.$ and $A_{S}$ belong to $\left.C(H)\right\}$. Since $\mathfrak{T}_{S} \subset \mathcal{J}_{S}$, we obtain that $\mathfrak{T}_{S} \subset \mathcal{F}_{S}$.

In Theorem 3.2(i) it was shown that $\check{\mathfrak{T}}_{S}$ is a closed subspace of $\widehat{T}(H)$. Since $\left(\mathfrak{T}_{S},|\cdot|_{2}\right)$ is isometrically isomorphic to $\check{\mathfrak{T}}_{S}$, it is a Banach space. 
Since the norms $|\cdot|_{1}$ and $|\cdot|_{2}$ are equivalent, $\left(\mathfrak{T}_{S},|\cdot|_{1}\right)$ is also a Banach space.

For $A, B \in \mathcal{A}_{S}$,

$$
\begin{aligned}
\left.(A B)_{S}\right|_{D(S)} & =\left.(S A B-A B S)\right|_{D(S)} \\
& =\left.[(S A-A S) B+A(S B-B S)]\right|_{D(S)}=\left.\left(A_{S} B+A B_{S}\right)\right|_{D(S)},
\end{aligned}
$$

so that

$$
(A B)_{S}=A_{S} B+A B_{S}
$$

Let $T \in \mathfrak{T}_{S}$ and $A \in \mathcal{A}_{S}$. Then $T, T_{S} \in T(H)$. Since $\mathfrak{T}_{S} \subset \mathcal{A}_{S}$ and $T(H)$ is a two-sided ideal of $B(H)$, it follows that $A T \in T(H) \cap \mathcal{A}_{S}$ and, by (3.12),

$$
(A T)_{S}=A_{S} T+A T_{S} \in T(H) .
$$

Therefore $A T \in \mathfrak{T}_{S}$. Making use of (3.11), we obtain that

$$
\begin{aligned}
|A T|_{2} & =\max \left(|A T|,\left|(A T)_{S}\right|\right) \leq \max \left(\|A\||T|,\left\|A_{S}\right\||T|+\|A\|\left|T_{S}\right|\right) \\
& \leq\left(\|A\|+\left\|A_{S}\right\|\right) \max \left(|T|,\left|T_{S}\right|\right)=\|A\|_{S}|T|_{2} .
\end{aligned}
$$

Similarly, $T A \in \mathfrak{T}_{S}$ and $|T A|_{2} \leq\|A\|_{S}|T|_{2}$. Thus $\left(\mathfrak{T}_{S},|\cdot|_{2}\right)$ is a two-sided Banach $\mathcal{A}_{S}$-module. Part (i) is proved.

From (i) and from the fact that $\mathfrak{T}_{S} \subseteq \mathcal{A}_{S}$, we have that $\mathfrak{T}_{S}$ is an algebra. We also have that $T^{*} \in \mathfrak{T}_{S}$ and, since $\mathbb{T}_{S}=T_{S}$, it follows from (3.3) that $\left(T^{*}\right)_{S}=-\left(T_{S}\right)^{*} \in T(H)$. Taking this and (3.11) into account, we obtain that

$$
\left|T^{*}\right|_{1}=\left|T^{*}\right|+\left|\left(T^{*}\right)_{S}\right|=\left|T^{*}\right|+\left|-\left(T_{S}\right)^{*}\right|=|T|+\left|T_{S}\right|=|T|_{1}
$$

and

$$
\begin{aligned}
|T R|_{1} & =|T R|+\left|(T R)_{S}\right|=|T R|+\left|T_{S} R+T R_{S}\right| \\
& \leq\|T\||R|+\left|T_{S}\right|\|R\|+\|T\|\left|R_{S}\right| \\
& \leq|T||R|+\left|T_{S}\right||R|+|T|\left|R_{S}\right| \leq|T|_{1}|R|_{1},
\end{aligned}
$$

for $T, R \in \mathfrak{T}_{S}$. Hence $\left(\mathfrak{T}_{S},|\cdot|_{1}\right)$ is a Banach *-algebra.

Clearly, $\mathfrak{T}_{S}$ is dense in $C(H)$. For $T, R \in \mathfrak{T}_{S}$, it follows from (3.11) that

$$
\begin{aligned}
|T R|_{1} & =|T R|+\left|(T R)_{S}\right|=|T R|+\left|T_{S} R+T R_{S}\right| \\
& \leq\|T\||R|+\left|T_{S}\right|\|R\|+\|T\|\left|R_{S}\right| \\
& \leq\|T\|\left(|R|+\left|R_{S}\right|\right)+\left(|T|+\left|T_{S}\right|\right)\|R\| \\
& =\|T\||R|_{1}+|T|_{1}\|R\| .
\end{aligned}
$$

Thus $\left(\mathfrak{T}_{S},|\cdot|_{1}\right)$ is a $\mathbf{D}$-subalgebra of $C(H)$ with the constant $D=1$.

If $S$ is selfadjoint, it follows from Theorem 3.2 that $\left(\check{\Phi}_{S}\right)^{\perp}=\widehat{\mathcal{A}}_{S}$ and

$$
\left(\widehat{\mathcal{F}}_{S}\right)^{\perp \perp}=\left(\check{\mathfrak{T}}_{S}\right)^{\perp} \subseteq\left(\check{\Phi}_{S}\right)^{\perp}=\widehat{\mathcal{A}}_{S}
$$


In order to prove that $\left(\widehat{\mathcal{F}}_{S}\right)^{\perp \perp}=\widehat{\mathcal{A}}_{S}$ it suffices to show that $\check{\Phi}_{S}$ is dense in $\check{\mathfrak{T}}_{S}$. For this we need the following lemma which is a partial case of the general result obtained by Gohberg and Krein [6, Theorem 6.3] for symmetrically normable ideals.

Lemma 3.6. Let $T \in T(H)$ and let $Q_{n}$ be finite rank projections which converge to $\mathbf{1}_{H}$ in the strong operator topology. Then

$$
\left|T-Q_{n} T\right| \rightarrow 0 \text { and }\left|T-T Q_{n}\right| \rightarrow 0 \text {, as } n \rightarrow \infty \text {. }
$$

Proof. Let $A=x \otimes y, x, y \in H$. By (3.5), $A^{*} A=\|y\|^{2}(x \otimes x)$ and the operator $\left(A^{*} A\right)^{1 / 2}=\frac{\|y\|}{\|x\|}(x \otimes x)$ has only one non-zero eigenvalue $\lambda=\|x\|\|y\|$. Hence

$$
|x \otimes y|=|A|=\operatorname{Tr}\left(A^{*} A\right)^{1 / 2}=\lambda=\|x\|\|y\| .
$$

If $T=\sum_{i=1}^{k} x_{i} \otimes y_{i}$ is a finite rank operator then, by (3.5) and (3.13),

$$
\begin{aligned}
\left|T-Q_{n} T\right| & =\left|\sum_{i=1}^{k} x_{i} \otimes\left(y_{i}-Q_{n} y_{i}\right)\right| \leq \sum_{i=1}^{k}\left|x \otimes\left(y_{i}-Q_{n} y_{i}\right)\right| \\
& =\sum_{i=1}^{k}\left\|x_{i}\right\|\left\|y_{i}-Q_{n} y_{i}\right\| \rightarrow 0,
\end{aligned}
$$

as $n \rightarrow \infty$. For any $T$ in $T(H)$ and any $\varepsilon>0$, there is a finite rank operator $T_{\varepsilon}$ such that $\left|T-T_{\varepsilon}\right|<\varepsilon$. Making use of the inequality (3.11), we obtain that

$$
\begin{aligned}
\left|T-Q_{n} T\right| & \leq\left|T-T_{\varepsilon}\right|+\left|T_{\varepsilon}-Q_{n} T_{\varepsilon}\right|+\left|Q_{n}\left(T-T_{\varepsilon}\right)\right| \\
& \leq \varepsilon+\left|T_{\varepsilon}-Q_{n} T_{\varepsilon}\right|+|| Q_{n} \|\left|T-T_{\varepsilon}\right| \\
& \leq 2 \varepsilon+\left|T_{\varepsilon}-Q_{n} T_{\varepsilon}\right| .
\end{aligned}
$$

Since $T_{\varepsilon}$ is a finite rank operator, by the above argument, there is $n_{\varepsilon}$ such that $\left|T_{\varepsilon}-Q_{n} T_{\varepsilon}\right| \leq \varepsilon$, for $n>n_{\varepsilon}$. Hence $\left|T-Q_{n} T\right| \leq 3 \varepsilon$ and $\left|T-Q_{n} T\right| \rightarrow 0$, as $n \rightarrow \infty$. Similarly, one can prove that $\left|T-T Q_{n}\right| \rightarrow 0$, as $n \rightarrow \infty$.

Proposition 3.7. Let $S$ be selfadjoint. Then $\Phi_{S}$ is dense in $\left(\mathfrak{T}_{S},|\cdot|_{1}\right)$.

Proof. Let $[S]$ be the selfadjoint operator constructed in Section 2. Then $D(S)=D([S])$, so that $\Phi_{S}=\Phi_{[S]}$. Since $B=S-[S]$ is a bounded operator, $B T-T B \in T(H)$, for $T \in T(H)$. Therefore, taking into account that

$$
(S T-T S)_{D(S)}=([S] T-T[S])_{D(S)}+(B T-T B)_{D(S)},
$$

we conclude that $\mathfrak{T}_{S}=\mathfrak{T}_{[S]}$ and $T_{S}=T_{[S]}+B T-T B$.

Making use of (3.11), we obtain that for any $T \in \mathfrak{T}_{S}$,

$$
\begin{aligned}
|T|+\left|T_{S}\right| & =|T|+\left|T_{[S]}+B T-T B\right| \\
& \leq|T|+\left|T_{[S]}\right|+2\|B\||T| \\
& \leq(1+2\|B\|)\left(|T|+\left|T_{[S]}\right|\right) .
\end{aligned}
$$


Similarly, $|T|+\left|T_{[S]}\right| \leq(1+2\|B\|)\left(|T|+\left|T_{S}\right|\right)$. Thus the norms $|\cdot|_{1}$ generated by the operators $S$ and $[S]$ on $\mathfrak{T}_{S}$ are equivalent. Hence to obtain the proof we only have to show that $\Phi_{[S]}$ is dense in $\left(\mathfrak{T}_{[S]},|\cdot|_{1}\right)$.

In every subspace $H_{S}(n)$ (see $(2.2)$ ) we choose an increasing sequence of finite-dimensional projections $\left\{Q_{n}^{k}\right\}_{k=1}^{\infty}$ converging to the projection $P_{S}(n)$ (see $(2.1))$ in the strong operator topology as $k \rightarrow \infty$. Set

$$
Q^{k}=\sum_{n=-k}^{k} \oplus Q_{n}^{k} .
$$

Then $Q^{k}$ are finite-dimensional projections commuting with $[S]$. Hence $Q^{k} \in \Phi_{[S]}$. The projections $Q^{k}$ converge to $\mathbf{1}_{H}$ in the strong operator topology. Let $T \in \mathfrak{T}_{[S]}$. Then $Q_{n} T \in \Phi_{[S]}$ and

$$
[S] Q^{k} T-Q^{k} T[S]=Q^{k}[S] T-Q^{k} T[S]=Q^{k}([S] T-T[S])=Q^{k} T_{[S]} .
$$

Therefore $\left(Q^{k} T\right)_{[S]}=Q^{k} T_{[S]}$.

Since $T, T_{[S]} \in T(H)$, we obtain from Lemma 3.6 that

$$
\left|T-Q^{k} T\right| \rightarrow 0 \text { and }\left|T_{[S]}-\left(Q^{k} T\right)_{[S]}\right|=\left|T_{[S]}-Q^{k} T_{[S]}\right| \rightarrow 0, \text { as } k \rightarrow \infty .
$$

Hence

$$
\left|T-Q^{k} T\right|_{1}=\left|T-Q^{k} T\right|+\left|T_{[S]}-\left(Q^{k} T\right)_{[S]}\right| \rightarrow 0
$$

as $k \rightarrow \infty$, so that $\Phi_{[S]}$ is dense in $\left(\mathfrak{T}_{[S]},|\cdot|_{1}\right)$.

Corollary 3.8. Let $S$ be a selfadjoint operator. Then:

(i) the Banach *-algebra $\left(\mathfrak{T}_{S},|\cdot|_{1}\right)$ is simple;

(ii) $\left(\check{\mathfrak{T}}_{S}\right)^{\perp}=\left(\check{\Phi}_{S}\right)^{\perp}=\widehat{\mathcal{A}}_{S}$;

(iii) the dual space of $\left(\mathfrak{T}_{S},|\cdot|_{2}\right)$ is isometrically isomorphic to the quotient space $\widehat{B}(H) / \widehat{\mathcal{A}}_{S}$.

Proof. Let $I$ be a closed two-sided ideal of $\left(\mathfrak{T}_{S},|\cdot|_{1}\right)$ and $0 \neq T \in I$. Since $D(S)$ is dense in $H$, there is $x \in D(S)$ such that $T x \neq 0$. Since $S$ is selfadjoint, it follows from the definition of $\mathfrak{T}_{S}$ that $T x \in D(S)$. From this and from the discussion before Lemma 3.1 we obtain that the rank one operators $y \otimes x$ and $T x \otimes z$ belong to $\mathfrak{T}_{S}$ for any $y, z \in D(S)$. By (3.5), $T(y \otimes x)=(y \otimes T x) \in I$ and

$$
(T x \otimes z)(y \otimes T x)=\|T x\|^{2}(y \otimes z) \in I .
$$

Thus $y \otimes z \in I$ and, therefore, $\Phi_{S} \subseteq I$. Since $I$ is closed, we obtain from Proposition 3.7 that $I=\mathfrak{T}_{S}$. Part (i) is proved.

Since the norms $|\cdot|_{1}$ and $|\cdot|_{2}$ are equivalent on $\mathfrak{T}_{S}$, it follows from Proposition 3.7 that $\Phi_{S}$ is dense in $\left(\mathfrak{T}_{S},|\cdot|_{2}\right)$. Taking into account that $\left(\mathfrak{T}_{S},|\cdot|_{2}\right)$ is isometrically isomorphic to the closed subspace $\check{\mathfrak{T}}_{S}$ of $\widehat{T}(H)$, 
we obtain that the linear subspace $\check{\Phi}_{S}$ is dense in $\check{\mathfrak{T}}_{S}$. From this and from Theorem 3.2(ii) we obtain $\left(\check{\mathfrak{T}}_{S}\right)^{\perp}=\left(\check{\Phi}_{S}\right)^{\perp}=\widehat{\mathcal{A}}_{S}$. Part (ii) is proved.

The dual space of $\left(\mathfrak{T}_{S},|\cdot|_{2}\right)$ is isometrically isomorphic to the dual space of the closed subspace $\check{\mathfrak{T}}_{S}$ of $\widehat{T}(H)$. Since $\widehat{T}(H)^{*}=\widehat{B}(H)$, part (iii) follows from (ii) and from Lemma 3.1.

Theorem 3.9. If $S$ is a selfadjoint operator then $\left(\widehat{\mathcal{F}}_{S}\right)^{\perp \perp}=\widehat{\mathcal{A}}_{S}$ and the second dual space of the algebra $\left(\mathcal{F}_{S},\|\cdot\|_{S}\right)$ is isometrically isomorphic to the algebra $\left(\mathcal{A}_{S},\|\cdot\|_{S}\right)$.

Proof. Combining Theorem 3.2(i) and Corollary 3.8(ii) yields $\left(\widehat{\mathcal{F}}_{S}\right)^{\perp \perp}=\widehat{\mathcal{A}}_{S}$. Therefore it follows from Lemma 3.1 that the second dual space of $\left(\widehat{\mathcal{F}}_{S},\|\cdot\|\right)$ is isometrically isomorphic to $\left(\widehat{\mathcal{A}}_{S},\|\cdot\|\right)$. Taking into account that $\left(\mathcal{F}_{S},\|\cdot\|_{S}\right)$ is isometrically isomorphic to $\left(\widehat{\mathcal{F}}_{S},\|\cdot\|\right)$ and that $\left(\mathcal{A}_{S},\|\cdot\|_{S}\right)$ is isometrically isomorphic to $\left(\widehat{\mathcal{A}}_{S},\|\cdot\|\right)$, we complete the proof.

\section{Isomorphism of the algebras $\mathcal{F}_{S}$ and $\mathcal{A}_{S}$.}

In this section we study the problem of classification of the algebras $\mathcal{F}_{S}$ and $\mathcal{A}_{S}$ up to ${ }^{*}$-isomorphism. For isometrical ${ }^{*}$-isomorphism this problem is completely solved in Theorem 4.4. As far as bounded but not necessarily isometrical *-isomorphism is concerned, we have obtained some partial results in Theorems 4.6 and 4.8 for the case when $\mathrm{S}$ is selfadjoint.

Banach *-algebras $\left(\mathcal{A},\|\|_{\mathcal{A}}\right)$ and $\left(\mathcal{B},\|\|_{\mathcal{B}}\right)$ are ${ }^{*}$-isomorphic if there is a bounded *-isomorphism $\varphi$ from $\mathcal{A}$ onto $\mathcal{B}$. They are isometrically *-isomorphic if, in addition, $\|\varphi(A)\|_{\mathcal{B}}=\|A\|_{\mathcal{A}}$, for $A \in \mathcal{A}$.

Let $\left(\mathcal{A},\|\|_{\mathcal{A}}\right)$ and $\left(\mathcal{B},\|\|_{\mathcal{B}}\right)$ be Banach *-algebras of operators on Hilbert spaces $H$ and $\mathcal{H}$ (the norms $\|\cdot\|_{\mathcal{A}}$ and $\|\cdot\|_{\mathcal{B}}$ do not, generally speaking, coincide with the operator norms in $B(H)$ and $B(\mathcal{H}))$ and let $\varphi$ be a bounded *-isomorphism from $\mathcal{A}$ onto $\mathcal{B}$. An isometry operator $U$ from $H$ into $\mathcal{H}$ implements $\varphi$ if

$$
\varphi(A)=U A U^{*}, \quad A \in \mathcal{A} .
$$

Lemma 4.1. Let $R$ and $T$ be symmetric operators on $\mathcal{H}, S$ be a symmetric operators on $H, U$ be an isometry operator from $\mathcal{H}$ onto $H$ and $t \in \mathbb{R}$.

(i) If $\mathcal{F}_{R}=\mathcal{F}_{T}$ then the norms $\|\cdot\|_{R}$ and $\|\cdot\|_{T}$ on this algebra are equivalent, so that the Banach ${ }^{*}$-algebras $\left(\mathcal{F}_{R},\|\cdot\|_{R}\right)$ and $\left(\mathcal{F}_{T},\|\cdot\|_{T}\right)$ are ${ }^{*}$-isomorphic.

(ii) If $R= \pm T+t \mathbf{1}_{\mathcal{H}}$ then $\mathcal{F}_{R}=\mathcal{F}_{T}$ and the norms $\|\cdot\|_{R}$ and $\|\cdot\|_{T}$ coincide.

(iii) If $S=\lambda U T U^{*}+B$, where $0 \neq \lambda \in R$ and $B$ is a bounded selfadjoint operator, then $A \rightarrow U A U^{*}$ is a bounded ${ }^{*}$-isomorphism from $\left(\mathcal{F}_{T},\|\cdot\|_{T}\right)$ onto $\left(\mathcal{F}_{S},\|\cdot\|_{S}\right)$. If $\lambda= \pm 1$ and $B=t \mathbf{1}_{H}$ then $A \rightarrow U A U^{*}$ is an isometric *-isomorphism. 
The same results hold for the algebras $\mathcal{A}_{S}$.

Proof. By Proposition $3.2[\mathbf{1 3}]$, the algebras $\mathcal{F}_{R}$ and $\mathcal{F}_{T}$ are semisimple. Hence if $\mathcal{F}_{R}=\mathcal{F}_{T}$ then it follows from Johnson's uniqueness of norm theorem that the norms $\|\cdot\|_{R}$ and $\|\cdot\|_{T}$ on this algebra are equivalent. Therefore the identity mapping is a bounded ${ }^{*}$-isomorphism from $\left(\mathcal{F}_{R},\|\cdot\|_{R}\right)$ onto $\left(\mathcal{F}_{T},\|\cdot\|_{T}\right)$.

Let $R= \pm T+t \mathbf{1}_{\mathcal{H}}$. Then $D(R)=D(T)$ and $A_{T}=A_{R}$ for any $A \in \mathcal{A}_{T}$. Hence $\|A\|_{R}=\|A\|_{T}$ and $\mathcal{A}_{R}=\mathcal{A}_{T}$. The sets of finite rank operators in the algebras $\mathcal{F}_{R}$ and $\mathcal{F}_{T}$ coincide and, since these algebras are the closures of these sets with respect to the norm $\|\cdot\|_{T}$, we obtain that $\mathcal{F}_{S}=\mathcal{F}_{T}$.

If $S=\lambda U T U^{*}+B$ then $D(S)=U D(T)$ and, for $A \in \mathcal{A}_{T}$,

$$
\begin{aligned}
U A U^{*} D(S) & =U A D(T) \subseteq U D(T)=D(S) \text { and } \\
S U A U^{*}-U A U^{*} S & =\lambda U(T A-A T) U^{*}+(B A-A B),
\end{aligned}
$$

so that $U A U^{*} \in \mathcal{A}_{S}$ and $\left(U A U^{*}\right)_{S}=\lambda U A_{T} U^{*}+(B A-A B)$. Thus $\mathcal{A}_{S}=U \mathcal{A}_{T} U^{*}$ and

$$
\begin{aligned}
\left\|U A U^{*}\right\|_{S} & =\left\|U A U^{*}\right\|+\left\|\left(U A U^{*}\right)_{S}\right\|=\|A\|+\left\|\lambda U A_{T} U^{*}+(B A-A B)\right\| \\
& \leq\|A\|+\lambda\|A\|+2\|B\|\|A\| \leq \max (\lambda, 1+2\|B\|)\|A\|_{T},
\end{aligned}
$$

so that $\psi(A)=U A U^{*}$ is a bounded *-isomorphism from $\left(\mathcal{A}_{T},\|\cdot\|_{T}\right)$ onto $\left(\mathcal{A}_{S},\|\cdot\|_{S}\right)$. If $A$ is a finite rank operator in $\mathcal{A}_{T}$ then $U A U^{*}$ is a finite rank operator in $\mathcal{A}_{S}$. Therefore $\mathcal{F}_{S}=\psi\left(\mathcal{F}_{T}\right)$.

Let $S$ be a symmetric operator with domain $D(S)$. It was shown in Lemma $3.1[\mathbf{1 3}]$ that a finite rank operator $A$ belongs to $\mathcal{F}_{S}$ if and only if

$$
A=\sum_{i=1}^{n} x_{i} \otimes y_{i}, \quad \text { where } x_{i}, y_{i} \in D(S) .
$$

Theorem 4.2. Let $S$ and $T$ be symmetric operators on $H$ and $\mathcal{H}$ and let $\mathcal{B}$ and $\mathcal{C}$ be closed ${ }^{*}$-subalgebras of $\left(\mathcal{A}_{S},\|\cdot\|_{S}\right)$ and $\left(\mathcal{A}_{T},\|\cdot\|_{T}\right)$, respectively, such that $\mathcal{F}_{S} \subseteq \mathcal{B}$ and $\mathcal{F}_{T} \subseteq \mathcal{C}$. Let $\psi$ be a bounded ${ }^{*}$-isomorphism from $\mathcal{C}$ onto $\mathcal{B}$ and let $\varphi=\psi \mid \mathcal{F}_{T}$. Then:

(i) $\varphi$ is a bounded ${ }^{*}$-isomorphism of $\left(\mathcal{F}_{T},\|\cdot\|_{T}\right)$ onto $\left(\mathcal{F}_{S},\|\cdot\|_{S}\right)$;

(ii) there is an isometry operator $U$ from $\mathcal{H}$ onto $H$ implementing $\psi$ :

$$
\psi(A)=U A U^{*}, \quad \text { for } A \in \mathcal{C},
$$

and $D(S)=U D(T)$ and $\mathcal{F}_{U T U^{*}}=\mathcal{F}_{S}$.

Proof. For $x, y \in D(T), x \neq 0, y \neq 0$, set $Y=\varphi(x \otimes y)$. If $Y$ is not a rank one operator, there are $z, u \in D(S)$ such that $Y z \neq 0, Y u \neq 0$ and $Y z \perp Y u$. Since $Y \in \mathcal{A}_{S}$, we have that $Y z, Y u \in D(S)$, so that $Y z \otimes z \in \mathcal{F}_{S}$ and $u \otimes Y u \in \mathcal{F}_{S}$. By $(3.5)$

$$
(Y z \otimes z)(u \otimes Y u)=(Y u, Y z)(u \otimes z)=0 .
$$


Since $(z \otimes z)^{*}=z \otimes z$ and $\varphi$ is a ${ }^{*}$-isomorphism, it follows from (3.5) that

$$
\begin{aligned}
\left(\varphi^{-1}(z \otimes z) x\right) \otimes y & =(x \otimes y)\left[\varphi^{-1}(z \otimes z)\right]^{*} \\
& =\varphi^{-1}(Y) \varphi^{-1}(z \otimes z)=\varphi^{-1}(z \otimes Y z) \neq 0 .
\end{aligned}
$$

Thus $\varphi^{-1}(z \otimes z) x \neq 0$. Similarly, $\varphi^{-1}(u \otimes u) x \neq 0$. From this and from (3.5) and (4.2) it follows that

$$
\begin{aligned}
0 & =\varphi^{-1}((Y z \otimes z)(u \otimes Y u))=\varphi^{-1}\left((z \otimes z) Y^{*} Y(u \otimes u)\right) \\
& =\varphi^{-1}(z \otimes z) \varphi^{-1}\left(Y^{*}\right) \varphi^{-1}(Y) \varphi^{-1}(u \otimes u) \\
& =\varphi^{-1}(z \otimes z)(y \otimes x)(x \otimes y) \varphi^{-1}(u \otimes u) \\
& =\varphi^{-1}(z \otimes z)\|y\|^{2}(x \otimes x) \varphi^{-1}(u \otimes u) \\
& =\|y\|^{2}\left(\left[\varphi^{-1}(u \otimes u) x\right] \otimes\left[\varphi^{-1}(z \otimes z) x\right]\right) \neq 0 .
\end{aligned}
$$

This contradiction shows that $Y$ is a rank one operator. Hence $Y \in \mathcal{F}_{S}$ and, by (4.1), $\varphi$ maps all finite rank operators in $\mathcal{F}_{T}$ into finite rank operators in $\mathcal{F}_{S}$. Since $\varphi$ is bounded $\varphi\left(\mathcal{F}_{T}\right) \subseteq \mathcal{F}_{S}$. Similarly, $\varphi^{-1}\left(\mathcal{F}_{S}\right) \subseteq \mathcal{F}_{T}$, so that $\varphi$ is a bounded ${ }^{*}$-isomorphism from $\mathcal{F}_{T}$ onto $\mathcal{F}$. Part (i) is proved.

Fix $x_{0} \in D(T),\left\|x_{0}\right\|=1$. Since $x_{0} \otimes x_{0}$ is a projection, $\varphi\left(x_{0} \otimes x_{0}\right)$ is a one-dimensional projection in $\mathcal{F}_{S}$. By (4.1), we can choose $\xi_{0}$ in $D(S)$, $\left\|\xi_{0}\right\|=1$, such that $\varphi\left(x_{0} \otimes x_{0}\right)=\xi_{0} \otimes \xi_{0}$. Let $y \in D(T)$. Making use of the equality $x_{0} \otimes y=\left(x_{0} \otimes y\right)\left(x_{0} \otimes x_{0}\right)$, we obtain that

$$
\begin{aligned}
\varphi\left(x_{0} \otimes y\right) & =\varphi\left(x_{0} \otimes y\right) \varphi\left(x_{0} \otimes x_{0}\right) \\
& =\varphi\left(x_{0} \otimes y\right)\left(\xi_{0} \otimes \xi_{0}\right)=\xi_{0} \otimes \varphi\left(x_{0} \otimes y\right) \xi_{0} .
\end{aligned}
$$

Since $\varphi\left(x_{0} \otimes y\right) \in \mathcal{F}_{S}$, it follows from (4.1) that $\varphi\left(x_{0} \otimes y\right) \xi_{0}$ belongs to $D(S)$.

Now $U: y \in D(T) \rightarrow \varphi\left(x_{0} \otimes y\right) \xi_{0}$ is a linear mapping from $D(T)$ into $D(S)$ and $\varphi\left(x_{0} \otimes y\right)=\xi_{0} \otimes U y$. Then

$$
\begin{aligned}
\varphi\left(\left(y \otimes x_{0}\right)\left(x_{0} \otimes y\right)\right) & =\|y\|^{2} \varphi\left(x_{0} \otimes x_{0}\right)=\|y\|^{2}\left(\xi_{0} \otimes \xi_{0}\right) \\
& =\varphi\left(\left(x_{0} \otimes y\right)^{*}\right) \varphi\left(x_{0} \otimes y\right) \\
& =\left(U y \otimes \xi_{0}\right)\left(\xi_{0} \otimes U y\right)=\|U y\|^{2}\left(\xi_{0} \otimes \xi_{0}\right) .
\end{aligned}
$$

Thus $\|U y\|^{2}=\|y\|^{2}$, for $y \in D(T)$, and $U$ extends to an isometry operator from $\mathcal{H}$ into $H$ which we also denote by $U$. We have that, for $x, y \in D(T)$,

$$
\begin{aligned}
\varphi(x \otimes y) & =\varphi\left(\left(x_{0} \otimes y\right)\left(x \otimes x_{0}\right)\right)=\left(\xi_{0} \otimes U y\right)\left(\xi_{0} \otimes U x\right)^{*} \\
& =U x \otimes U y=U(x \otimes y) U^{*} .
\end{aligned}
$$

Similarly, there is an isometry operator $V$ which maps $D(S)$ into $D(T)$ such that $\varphi^{-1}(\xi \otimes \eta)=V \xi \otimes V \eta$, for $\xi, \eta \in D(S)$. Hence

$$
\xi \otimes \eta=\varphi\left(\varphi^{-1}(\xi \otimes \eta)\right)=\varphi(V \xi \otimes V \eta)=U V \xi \otimes U V \eta .
$$


Thus $U V \xi=\lambda(\xi) \xi$ where $\lambda$ is a function on $D(S)$ such that $|\lambda(\xi)|=1$. Hence $U D(T)=D(S)$. Since $D(S)$ is dense in $H$ and $U$ is an isometry operator, we have $U \mathcal{H}=H$.

Let $A \in \mathcal{C}$ and set $R=U^{*} \psi(A) U$. Then $x \otimes y \in \mathcal{F}_{T}$, for any $x, y \in D(T)$, and, since $\mathcal{F}_{T}$ is an ideal of $\mathcal{A}_{T}$, we have $A(x \otimes y)=x \otimes A y \in \mathcal{F}_{T}$. By (4.3),

$$
\begin{aligned}
R(x \otimes y) & =U^{*} \psi(A) U(x \otimes y)=U^{*} \psi(A) U(x \otimes y) U^{*} U \\
& =U^{*} \psi(A) \varphi(x \otimes y) U=U^{*} \psi(A) \psi(x \otimes y) U \\
& =U^{*} \psi(A(x \otimes y)) U=U^{*} \varphi(x \otimes A y) U=x \otimes A y .
\end{aligned}
$$

Therefore $R(x \otimes y)=x \otimes R y=x \otimes A y$, so that $R y=A y$. Thus $R=A$ and

$$
\psi(A)=U A U^{*} \text {. }
$$

The operator $F=U T U^{*}$ is symmetric and $D(F)=U D(T)=D(S)$. By Lemma 4.1, $\mathcal{F}_{F}=U \mathcal{F}_{T} U^{*}$ and $A \rightarrow U A U^{*}$ is an isometric *-isomorphism from $\left(\mathcal{F}_{T},\|\cdot\|_{T}\right)$ onto $\left(\mathcal{F}_{F},\|\cdot\|_{F}\right)$. Hence

$$
\varphi\left(U^{*} B U\right)=U\left(U^{*} B U\right) U^{*}=B, \quad \text { for } B \in \mathcal{F}_{F},
$$

is a bounded ${ }^{*}$-isomorphism from $\mathcal{F}_{F}$ onto $\mathcal{F}_{S}$. Therefore $\mathcal{F}_{F}=\mathcal{F}_{S}$.

It was shown in Theorem $3.4[\mathbf{1 3}]$ that the algebra $\left(\mathcal{F}_{S},\|\cdot\|_{S}\right)$ has a bounded approximate identity if and only if $S$ is selfadjoint. Making use of this and of Theorem 4.2, we obtain the following result.

Corollary 4.3. If the algebras $\mathcal{F}_{S}$ and $\mathcal{F}_{T}$ are ${ }^{*}$-isomorphic or the algebras $\mathcal{A}_{S}$ and $\mathcal{A}_{T}$ are ${ }^{*}$-isomorphic then the operators $S$ and $T$ are either selfadjoint or non-selfadjoint at the same time.

Apart from the sufficient conditions of Lemma 4.1 and the necessary conditions of Corollary 4.3 for two algebras $\mathcal{F}_{S}$ and $\mathcal{F}_{T}$ to be ${ }^{*}$-isomorphic we do not know any other sufficient or necessary condition in the case when $S$ and $T$ are arbitrary symmetric operators. Later, in Theorem 4.6 and Corollary 4.8 we consider a particular case when the operators $S$ and $T$ are selfadjoint.

It follows from Theorem 4.2 that if $\mathcal{F}_{S}$ and $\mathcal{F}_{T}$ are ${ }^{*}$-isomorphic, they are unitary isomorphic. This, however, does not necessarily imply that they are isometrically isomorphic. In the following theorem we obtain necessary and sufficient conditions for algebras $\mathcal{F}_{S}$ and $\mathcal{F}_{T}$ to be isometrically ${ }^{*}$-isomorphic.

Theorem 4.4. The algebras $\left(\mathcal{F}_{S},\|\cdot\|_{S}\right)$ and $\left(\mathcal{F}_{T},\|\cdot\|_{T}\right)$ are isometrically *-isomorphic if and only if there are $\lambda \in \mathbb{R}$ and an isometry operator $U$ such that $S-\lambda \mathbf{1}_{H}= \pm U T U^{*}$. The same result holds for $\left(\mathcal{A}_{S},\|\cdot\|_{S}\right)$ and $\left(\mathcal{A}_{T},\|\cdot\|_{T}\right)$.

Proof. From Lemma 4.1 it follows that the conditions of the theorem are sufficient. From Theorem 4.2 it follows that if these conditions are necessary for the algebras $\left(\mathcal{F}_{S},\|\cdot\|_{S}\right)$ and $\left(\mathcal{F}_{T},\|\cdot\|_{T}\right)$ to be isometrically ${ }^{*}$-isomorphic, they are also necessary for the algebras $\left(\mathcal{A}_{S},\|\cdot\|_{S}\right)$ and $\left(\mathcal{A}_{T},\|\cdot\|_{T}\right)$. 
Let $\varphi$ be an isometric ${ }^{*}$-isomorphism from $\left(\mathcal{F}_{T},\|\cdot\|_{T}\right)$ onto $\left(\mathcal{F}_{S},\|\cdot\|_{S}\right)$ and let $U$ be the isometry operator as in Theorem 4.2 which implements $\varphi$ :

$$
\varphi(A)=U A U^{*}, \quad \text { for } A \in \mathcal{F}_{T} .
$$

Set $F=U T U^{*}$. Then $F$ is a symmetric operator on $H, D(S)=D(F)=$ $U D(T)$ and $\mathcal{F}_{S}=\mathcal{F}_{F}$. Since $\varphi$ is isometric, the norms $\|\cdot\|_{S}$ and $\|\cdot\|_{F}$ coincide.

We will show that there is $\lambda \in \mathbb{R}$ such that either $S-\lambda \mathbf{1}_{H}=F$ or $S-\lambda \mathbf{1}_{H}=-F$.

Step 1. Suppose that $z \in D(S)$ is not an eigenvector of $S$ and $\|z\|=1$. Set

$$
s=(S z, z), \quad t=(F z, z), \quad R=S-s \mathbf{1}_{H} \quad \text { and } G=F-t \mathbf{1}_{H} .
$$

Since $S$ an $F$ are symmetric, $s, t \in \mathbb{R}$, the operators $R$ and $G$ are symmetric and

$$
D(R)=D(G), \quad R z \neq 0 \quad \text { and }(R z, z)=(G z, z)=0 .
$$

Set $D=D(R)=D(G)$. Since $\mathcal{F}_{S}=\mathcal{F}_{F}$ and the norms $\|\cdot\|_{S}$ and $\|\cdot\|_{F}$ coincide, it follows from Lemma 4.1 that $\mathcal{F}_{R}=\mathcal{F}_{G}$ and the norms $\|\cdot\|_{R}$ and $\|\cdot\|_{G}$ coincide.

Taking into account that $R$ and $G$ are symmetric, we obtain from (3.5) that

$$
\begin{aligned}
\|y \otimes x\|_{R} & =\|y \otimes x\|+\|y \otimes R x-(R y) \otimes x\|=\|y \otimes x\|_{G} \\
& =\|y \otimes x\|+\|y \otimes G x-(G y) \otimes x\|,
\end{aligned}
$$

for $x, y \in D$. Therefore

$$
\|y \otimes R x-(R y) \otimes x\|=\|y \otimes G x-(G y) \otimes x\| .
$$

Represent the elements $R x$ and $G x$ in the form

$$
R x=\alpha(x) x+x_{R} \text { and } G x=\beta(x) x+x_{G},
$$

where $x_{R}$ and $x_{G}$ are orthogonal to $x$. Then

$$
\alpha(x)\|x\|^{2}=(R x, x)=(x, R x)=\overline{\alpha(x)}\|x\|^{2} .
$$

Thus $\alpha(x)$ is real, for $x \in D$. Therefore

$$
\begin{aligned}
x \otimes R x-(R x) \otimes x & =\alpha(x)(x \otimes x)+x \otimes x_{R}-\alpha(x)(x \otimes x)-x_{R} \otimes x \\
& =x \otimes x_{R}-x_{R} \otimes x .
\end{aligned}
$$

Since $x$ and $x_{R}$ are orthogonal, any $u \in H$ can be represented in the form $u=\nu x+\tau x_{R}+\widetilde{u}$, where $\nu, \tau \in \mathbb{C}$ and $\widetilde{u}$ is orthogonal to $x$ and $x_{R}$. Therefore

$$
\|u\|=|\nu|^{2}\|x\|^{2}+|\tau|^{2}\|x\|^{2}+\|\widetilde{u}\|^{2}
$$


and, by (3.5),

$$
\begin{aligned}
\left\|\left(x \otimes x_{R}+x_{R} \otimes x\right) u\right\|^{2} & =\left\|(u, x) x_{R}+\left(u, x_{R}\right) x\right\|^{2} \\
& =\|\nu\| x\left\|^{2} x_{R}+\tau\right\| x_{R}\left\|^{2} x\right\|^{2} \\
& =|\nu|^{2}\|x\|^{4}\left\|x_{R}\right\|^{2}+|\tau|^{2}\left\|x_{R}\right\|^{4}\|x\|^{2} \\
& =\|x\|^{2}\left\|x_{R}\right\|^{2}\left(|\nu|^{2}\|x\|^{2}+|\tau|^{2}\left\|x_{R}\right\|^{2}\right) .
\end{aligned}
$$

Consequently,

$$
\|x \otimes R x-(R x) \otimes x\|^{2}=\left\|x \otimes x_{R}-x_{R} \otimes x\right\|^{2}=\|x\|^{2}\left\|x_{R}\right\|^{2} .
$$

Similarly, $\|x \otimes G x-(G x) \otimes x\|^{2}=\|x\|^{2}\left\|x_{G}\right\|^{2}$ and it follows from (4.5) that

$$
\left\|x_{R}\right\|=\left\|x_{G}\right\|, \quad \text { for } x \in D .
$$

Therefore we obtain from (4.6) that for $x \in D$

$$
\begin{aligned}
\|x\|^{2}\|R x\|^{2}-|(R x, x)|^{2} & =\|x\|^{2}\left(|\alpha(x)|^{2}\|x\|^{2}+\left\|x_{R}\right\|^{2}\right)-|\alpha(x)|^{2}\|x\|^{4} \\
& =\|x\|^{2}\left\|x_{R}\right\|^{2}=\|x\|^{2}\left\|x_{G}\right\|^{2} \\
& =\|x\|^{2}\|G x\|^{2}-|(G x, x)|^{2} .
\end{aligned}
$$

Hence

$$
\|x\|^{2}\left(\|R x\|^{2}-\|G x\|^{2}\right)=|(R x, x)|^{2}-|(G x, x)|^{2} .
$$

In particular, it follows from (4.4), (4.6) and (4.7) that

$$
R z=z_{R}, \quad G z=z_{G} \text { and }\|R z\|=\|G z\| .
$$

Step 2. Set $D_{Z}^{\perp}=\{y \in D: y$ is orthogonal to $z\}$. Let $y \in D_{Z}^{\perp}$ and $x=y+\mu z$, $\mu \in \mathbb{C}$. Then $\|x\|^{2}=\|y\|^{2}+\|\mu z\|^{2}=\|y\|^{2}+|\mu|^{2}$ and, by (4.8),

$$
\begin{aligned}
\|R x\|^{2}-\|G x\|^{2}= & \|R y\|^{2}+\|\mu R z\|^{2}+2 \operatorname{Re}[\mu(R z, R y)] \\
& -\|G y\|^{2}-\|\mu G z\|^{2}-2 \operatorname{Re}[\mu(G z, G y)] \\
= & A+2 \operatorname{Re}(\mu B),
\end{aligned}
$$

where

$$
A=\|R y\|^{2}-\|G y\|^{2} \quad \text { and } \quad B=(R z, R y)-(G z, G y) .
$$

Since $R$ is symmetric, it follows from (4.4) that

$$
\begin{aligned}
(R x, x) & =(R y, y)+(\mu R z, y)+(R y, \mu z)+(\mu R z, \mu z) \\
& =(R y, y)+2 \operatorname{Re}[\mu(R z, y)] .
\end{aligned}
$$

Similarly, $(G x, x)=(G y, y)+2 \operatorname{Re}[\mu(G z, y)]$.

Let $\mu=r e^{i \psi}$. Substituting all this in (4.7), we obtain that

$$
\begin{aligned}
& \left(\|y\|^{2}+r^{2}\right)\left[A+2 r \operatorname{Re}\left(e^{i \psi} B\right)\right] \\
& =\left\{(R y, y)+2 r \operatorname{Re}\left[e^{i \psi}(R z, y)\right]\right\}^{2}-\left\{(G y, y)+2 r \operatorname{Re}\left[e^{i \psi}(G z, y)\right]\right\}^{2} .
\end{aligned}
$$


Set

$$
\begin{aligned}
& C=(R y, y) \operatorname{Re}\left[e^{i \psi}(R z, y)\right]-(G y, y) \operatorname{Re}\left[e^{i \psi}(G z, y)\right] \quad \text { and } \\
& E=\left\{\operatorname{Re}\left[e^{i \psi}(R z, y)\right]\right\}^{2}-\left\{\operatorname{Re}\left[e^{i \psi}(G z, y)\right]\right\}^{2} .
\end{aligned}
$$

Since $R$ and $G$ are symmetric, $(R y, y)$ and $(G y, y)$ are real. Hence

$$
C=\operatorname{Re}\left\{e^{i \psi}[(R y, y)(R z, y)-(G y, y)(G z, y)]\right\} .
$$

Comparing the coefficients of the same powers of $r$ in (4.9), we obtain that

$$
\operatorname{Re}\left(e^{i \psi} B\right)=0, \quad A=4 E \text { and } C=0 .
$$

Taking into account that $\operatorname{Re}\left(e^{i \psi} K\right)=0$, for $0 \leq \psi<2 \pi$, implies $K=0$, we obtain that $C=0$ implies

$$
(R y, y)(R z, y)-(G y, y)(G z, y)=0 .
$$

Set $(R z, y)=a e^{i b}$ and $(G z, y)=c e^{i d}$. Then

$$
\begin{aligned}
E & =a^{2}\left[\operatorname{Re}\left(e^{i(\psi+b)}\right)\right]^{2}-c^{2}\left[\operatorname{Re}\left(e^{i(\psi+d)}\right)\right]^{2} \\
& =a^{2} \cos ^{2}(\psi+b)-c^{2} \cos ^{2}(\psi+d) .
\end{aligned}
$$

Since $A=4 E$ and since $A$ does not depend on $\psi$, neither does $E$. Hence $a^{2}=c^{2}$ and $d=b$ or $d=b+\pi$. Since $a \geq 0$ and $c \geq 0, a=c$. Thus

$$
(R z, y)= \pm(G z, y), \quad \text { for } y \in D_{Z}^{\perp} \text {. }
$$

Since $D$ is dense in $\mathcal{H}, D_{Z}^{\perp}$ is dense in the subspace $\{\mathbb{C} z\}^{\perp}$. Hence (4.11) holds for all $y \in\{\mathbb{C} z\}^{\perp}$. From (4.9) it follows that $R z=z_{R} \in\{\mathbb{C} z\}^{\perp}$. Substituting $R z$ for $y$ in (4.11), we obtain $\|R z\|=(R z, R z)= \pm(G z, R z)$. Let $G z=\nu R z+u$, where $\nu \in \mathbb{C}$ and $u$ is orthogonal to $R z$. Then

$$
\|R z\|^{2}= \pm(G z, R z)= \pm \nu\|R z\|^{2} .
$$

Since $R z \neq 0$ (see (4.4)), $\nu= \pm 1$. Taking (4.9) into account, we obtain

$$
\begin{aligned}
\|R z\|^{2} & =\|G z\|^{2}=(\nu R z+u, \nu R z+u) \\
& =|\nu|^{2}\|R z\|^{2}+\|u\|^{2}=\|R z\|^{2}+\|u\|^{2} .
\end{aligned}
$$

Hence $u=0$ and either $R z=G z$ or $R z=-G z$.

Step 3. Let $R z=G z$. Set $W=R-G$. Then $W$ is symmetric, $W z=0$ and it follows from (4.10) that

$$
[(R y, y)-(G y, y)](R z, y)=(W y, y)(R z, y)=0, \quad \text { for } y \in D_{Z}^{\perp} .
$$

Any $x \in D$ can be represented in the form $x=y+\mu z$ where $\mu \in \mathbb{C}$ and $y \in D_{Z}^{\perp}$. Then $W x=W y$ and, since $(R z, z)=0$, we have $(R z, x)=(R z, y)$. Since $W z=0$,

$$
\begin{aligned}
(W x, x)(R z, x) & =(W y, y+\mu z)(R z, y) \\
& =[(W y, y)+(y, \mu W z)](R z, y)=(W y, y)(R z, y)=0 .
\end{aligned}
$$


Therefore

$$
(W x, x)(R z, x)=0, \quad \text { for } x \in D .
$$

Let $X=\{x \in H:(R z, x)=0\}$ be the orthogonal complement of the subspace $\{\mathbb{C} R z\}$ in $H$. By $(4.4), R z \neq 0$, so $X$ has codimension 1. Set $\mathcal{D}=\{x \in D: x \notin X\}$. Since $D$ is dense in $H, \mathcal{D}$ is also dense in $H$. For $x \in \mathcal{D}$, we have $(R z, x) \neq 0$. Hence, by (4.12),

$$
(W x, x)=0 .
$$

If $x, y \in \mathcal{D}$, there is $r>0$ such that $x+r e^{i \psi} y \in \mathcal{D}$, for all $0 \leq \psi<2 \pi$. Taking into account that $W$ is symmetric, we obtain that

$$
\begin{aligned}
0 & =\left(W\left(x+r e^{i \psi} y\right), x+r e^{i \psi} y\right)=(W x, x)+2 r \operatorname{Re}\left[e^{i \psi}(W y, x)\right]+r^{2}(W y, y) \\
& =2 r \operatorname{Re}\left[e^{i \psi}(W y, x)\right] .
\end{aligned}
$$

Hence $(W y, x)=0$. Since $\mathcal{D}$ is dense in $H$, we have $W y=0$, for $y \in \mathcal{D}$.

Let $u \in D \cap X$, so that $(R z, u)=0$. For $y \in \mathcal{D},(R z, y+u)=(R z, y) \neq 0$. Hence $y+u \in \mathcal{D}$ and $0=W(y+u)=W y+W u=W u$. Thus $W x=0$, for all $x \in D$, so that $R=G$. Hence $S-s \mathbf{1}_{H}=F-t \mathbf{1}_{H}$. Setting $\lambda=s-t$, we obtain that

$$
S-\lambda \mathbf{1}_{H}=F=U T U^{*} .
$$

Similarly, in the case when $R z=-G z$ we obtain that $S-\lambda \mathbf{1}_{H}=-F=$ $-U T U^{*}$ which concludes the proof of the theorem.

In the rest of this section we study conditions for the algebras $\mathcal{F}_{S}$ and $\mathcal{F}_{T}$ to be ${ }^{*}$-isomorphic but not necessarily isometrically ${ }^{*}$-isomorphic in the case when $S$ and $T$ are selfadjoint operators. Taking Theorem 4.2(ii) into account, we may assume, without loss of generality, that $\mathcal{F}_{S}=\mathcal{F}_{T}$ and $D(S)=D(T)$.

In Example 4.7 we show that the coincidence of the domains of selfadjoint operators $S$ and $T$ even in the case when $\operatorname{Sp}(S) \subseteq \mathbb{Z}, \operatorname{Sp}(T) \subseteq \mathbb{Z}$ and $S$ and $T$ have the same sets of eigenvectors is not sufficient for $\mathcal{F}_{S}=\mathcal{F}_{T}$. In other words, the algebras $\mathcal{F}_{S}$ and $\mathcal{F}_{T}$ may be the closures of the same set of finite rank operators and, nevertheless, be non-isomorphic. Necessary and sufficient conditions for these algebras to be ${ }^{*}$-isomorphic will be obtained in Theorem 4.6.

Let $\mathfrak{H}$ be a Hilbert space with an orthogonal basis $\left\{e_{i}\right\}_{i=-\infty}^{\infty}$. Every operator $T$ in $B(\mathfrak{H})$ has a matrix representation $T=\left(t_{i j}\right),-\infty<i, j<\infty$, where $t_{i j}=\left(T e_{j}, e_{i}\right)$. A matrix $M=\left(m_{i j}\right),-\infty<i, j<\infty$, is called a Schur multiplier, if, for any $T=\left(t_{i j}\right) \in B(\mathfrak{H})$, the matrix $M \circ T=\left(m_{i j} t_{i j}\right)$ belongs to $B(\mathfrak{H})$. Then $T \rightarrow M \circ T$ is a bounded map of $B(\mathfrak{H})$ into itself; it will also be denoted by $M$ and its norm by $|M|_{B(\mathfrak{H})}$. 
Let $H=\sum_{i=-\infty}^{\infty} \oplus H_{i}$ be an orthogonal sum of Hilbert spaces $H_{i}$. Every operator $A$ in $B(H)$ has a block-matrix representation $A=\left(A_{i j}\right)$, $-\infty<i, j<\infty$, where $A_{i j}$ are bounded operators from $H_{j}$ into $H_{i}$.

Lemma 4.5. Let $M=\left(m_{i j}\right)$ be a Schur multiplier on $\mathfrak{H}$. It defines a bounded operator $\mathcal{M}$ on $B(H)$ by the formula

$$
\mathcal{M} \times A=\left(m_{i j} A_{i j}\right), \text { where } A=\left(A_{i j}\right) \in B(H),
$$

and $|\mathcal{M}|_{B(H)}=|M|_{B(\mathcal{H})}$.

Proof. Let $G=\left\{g_{j}\right\}_{j=-\infty}^{\infty}$ and $F=\left\{f_{j}\right\}_{j=-\infty}^{\infty}$ be sequences of elements such that $g_{j}, f_{j} \in H_{j}$ and $\left\|g_{j}\right\|=\left\|f_{j}\right\|=1$. For $A=\left(A_{i j}\right) \in B(H)$, let $T^{G, F}(A)=\left(a_{i j}^{G F}\right),-\infty<i, j<\infty$, be the matrix such that

$$
a_{i j}^{G F}=\left(A_{i j} g_{j}, f_{i}\right) \in \mathbb{C} .
$$

For $\alpha=\sum_{j=-\infty}^{\infty} \oplus \alpha_{j} e_{j} \in \mathfrak{H}$ and $\beta=\sum_{j=-\infty}^{\infty} \oplus \beta_{j} e_{j} \in \mathfrak{H}$, set

$$
x_{\alpha}^{G}=\sum_{j=-\infty}^{\infty} \oplus \alpha_{j} g_{j} \quad \text { and } \quad y_{\beta}^{F}=\sum_{j=-\infty}^{\infty} \oplus \beta_{j} f_{j} .
$$

Then $x_{\alpha}^{G}, y_{\beta}^{F} \in H,\left\|x_{\alpha}^{G}\right\|=\|\alpha\|,\left\|y_{\beta}^{F}\right\|=\|\beta\|$ and

$$
\begin{aligned}
\left(A x_{\alpha}^{G}, y_{\beta}^{F}\right) & =\sum_{i=-\infty}^{\infty} \sum_{j=-\infty}^{\infty} \alpha_{j} \bar{\beta}_{i}\left(A_{i j} g_{j}, f_{i}\right) \\
& =\sum_{i=-\infty}^{\infty} \sum_{j=-\infty}^{\infty} \alpha_{j} \bar{\beta}_{i} a_{i j}^{G F}=\left(T^{G, F}(A) \alpha, \beta\right) .
\end{aligned}
$$

Therefore $T^{G, F}(A) \in B(\mathfrak{H})$ and

$$
\begin{aligned}
\|A\| & =\sup _{\alpha, \beta, G, F} \frac{\left|\left(A x_{\alpha}^{G}, y_{\beta}^{F}\right)\right|}{\left\|x_{\alpha}^{G}\right\|\left\|y_{\beta}^{F}\right\|} \\
& =\sup _{G, F}\left(\sup _{\alpha, \beta} \frac{\left|\left(T^{G, F}(A) \alpha, \beta\right)\right|}{\|\alpha\|\|\beta\|}\right)=\sup _{G, F}\left\|T^{G, F}(A)\right\| .
\end{aligned}
$$

It follows from (4.13) that $T^{G, F}(\mathcal{M} \times A)=M \circ T^{G, F}(A)$. Since $M$ is a Schur multiplier, $M \circ T^{G, F}(A) \in B(\mathfrak{H})$ and, by (4.14),

$$
\begin{aligned}
\|\mathcal{M} \times A\| & =\sup _{G, F}\left\|T^{G, F}(\mathcal{M} \times A)\right\|=\sup _{G, F}\left\|M \circ T^{G, F}(A)\right\| \\
& \leq \sup _{G, F}|M|_{B(\mathfrak{H})}\left\|T^{G, F}(A)\right\|=|M|_{B(\mathfrak{H})} \sup _{G, F}\left\|T^{G, F}(A)\right\| \\
& =|M|_{B(\mathfrak{H})}\|A\| .
\end{aligned}
$$


Hence $|\mathcal{M}|_{B(H)} \leq|M|_{B(\mathfrak{H})}$. On the other hand, it is easy to see that $|M|_{B(\mathfrak{H})} \leq|\mathcal{M}|_{B(H)}$. Thus $|\mathcal{M}|_{B(H)}=|M|_{B(\mathfrak{H})}$.

Let $S$ and $T$ be selfadjoint operators on $H$ and assume that $\operatorname{Sp}(S) \subseteq \mathbb{Z}$, $\operatorname{Sp}(T) \subseteq \mathbb{Z}$ and that

$$
\begin{aligned}
H=\sum_{i=-\infty}^{\infty} \oplus H_{i} \quad \text { where }\left.S\right|_{H_{i}}=s_{i} \mathbf{1}_{H_{i}},\left.\quad T\right|_{H_{i}} & =t_{i} \mathbf{1}_{H_{i}}, \\
& s_{i} \neq s_{j} \text { and } t_{i} \neq t_{j} \text { if } i \neq j .
\end{aligned}
$$

Set

$$
\begin{aligned}
& M=\left(m_{i j}\right) \text { where } m_{i j}=\frac{s_{i}-s_{j}}{t_{i}-t_{j}}, \text { for } i \neq j, \text { and } m_{i i}=0, \text { and } \\
& N=\left(n_{i j}\right) \text { where } n_{i j}=\frac{t_{i}-t_{j}}{s_{i}-s_{j}}, \text { for } i \neq j, \text { and } n_{i i}=0 .
\end{aligned}
$$

Theorem 4.6. $\mathcal{F}_{S}=\mathcal{F}_{T}$ if and only if $M$ and $N$ are Schur multipliers.

Proof. In every $H_{i}$ we choose a non-decreasing sequence of finite-dimensional projections $\left\{Q_{i}^{p}\right\}_{p=1}^{\infty}$ which converge to $\mathbf{1}_{H_{i}}$ in the strong operator topology as $p \rightarrow \infty$. Set $Q_{p}=\sum_{i=-p}^{p} \oplus Q_{i}^{p}$. The finite-dimensional projections $Q_{p}$ commute with $S$ and $T$, belong to $\mathcal{F}_{S} \cap \mathcal{F}_{T}$ and converge to $\mathbf{1}_{H}$ in the strong operator topology. Therefore $\left\|Q_{p}\right\|_{S}=\left\|Q_{p}\right\|_{T}=\left\|Q_{p}\right\|=1$.

For any $A=\left(A_{i j}\right) \in \mathcal{A}_{S} \cap \mathcal{A}_{T}$,

$$
A_{S}=S A-A S=\left(A_{i j}^{S}\right) \text { and } A_{T}=T A-A T=\left(A_{i j}^{T}\right),
$$

where $A_{i j}^{S}=\left(s_{i}-s_{j}\right) A_{i j}$ and $A_{i j}^{T}=\left(t_{i}-t_{j}\right) A_{i j}$. Set $B=A_{T}$. Then $A_{S}=$ $\mathcal{M} \times B$

$$
\begin{aligned}
& \|A\|_{S}=\|A\|+\left\|A_{S}\right\|=\|A\|+\|\mathcal{M} \times B\| \quad \text { and } \\
& \|A\|_{T}=\|A\|+\left\|A_{T}\right\|=\|A\|+\|B\| .
\end{aligned}
$$

We assume now that $M$ and $N$ are Schur multipliers and show that $\mathcal{F}_{S}=$ $\mathcal{F}_{T}$. By Lemma 4.5 and (4.15),

$$
\begin{aligned}
\|A\|_{S} & \leq\|A\|+|M|\|B\| \\
& \leq\|A\|+|M|\left(\|A\|_{T}-\|A\|\right) \leq(|M|+1)\|A\|_{T} .
\end{aligned}
$$

Similarly,

$$
\|A\|_{T} \leq(|N|+1)\|A\|_{S} .
$$

Let $A \in \mathcal{F}_{S}$. Then $Q_{p} A \in \mathcal{F}_{S}$ and, since $Q_{p}$ commute with $S$,

$$
\left(Q_{p} A\right)_{S}=\text { Closure }\left(S Q_{p} A-Q_{p} A S\right)=\text { Closure } Q_{p}(S A-A S)=Q_{p} A_{S} .
$$

Since $A$ and $A_{S}$ are compact and since $Q_{p}$ converge to $\mathbf{1}_{H}$ in the strong operator topology, $\left\|A-Q_{p} A\right\| \rightarrow 0$ and $\left\|A_{S}-\left(Q_{p} A\right)_{S}\right\|=\left\|A_{S}-Q_{p} A_{S}\right\| \rightarrow 0$, as $p \rightarrow \infty$. 
Hence $\left\|A-Q_{p} A\right\|_{S} \rightarrow 0$, so that $\left\{Q_{p}\right\}$ is a bounded approximate identity in $\mathcal{F}_{S}$. Similarly, it is a bounded approximate identity in $\mathcal{F}_{T}$.

Let $A \in \mathcal{F}_{S}$. For any $p, Q_{p} T=Q_{p} T Q_{p}=T Q_{p}$ is a finite rank operator. Hence

$$
\left(Q_{p} A Q_{p}\right)_{T}=T\left(Q_{p} A Q_{p}\right)-\left(Q_{p} A Q_{p}\right) T=\left(T Q_{p}\right) A Q_{p}-Q_{p} A\left(Q_{p} T\right)
$$

is a finite rank operator. Therefore $Q_{p} A Q_{p} \in \mathcal{F}_{S} \cap \mathcal{F}_{T}$ and, by (4.17),

$$
\left\|Q_{p+k} A Q_{p+k}-Q_{p} A Q_{p}\right\|_{T} \leq(|N|+1)\left\|Q_{p+k} A Q_{p+k}-Q_{p} A Q_{p}\right\|_{S} .
$$

Since $\left\{Q_{p}\right\}$ is a bounded approximate identity in $\mathcal{F}_{S}$, the operators $Q_{p} A Q_{p}$ converge to $A$ with respect to $\|\cdot\|_{S}$. From the above inequality it follows that $\left\{Q_{p} A Q_{p}\right\}$ is a fundamental sequence with respect to $\|\cdot\|_{T}$. Hence there is $A_{1} \in \mathcal{F}_{T}$ such that $\left\|A_{1}-Q_{p} A Q_{p}\right\|_{T} \rightarrow 0$, as $p \rightarrow \infty$. Since $\left\|A-Q_{p} A Q_{p}\right\| \leq$ $\left\|A-Q_{p} A Q_{p}\right\|_{S} \rightarrow 0$ and $\left\|A_{1}-Q_{p} A Q_{p}\right\| \leq\left\|A_{1}-Q_{p} A Q_{p}\right\|_{T} \rightarrow 0$, as $p \rightarrow \infty$, we obtain that $A=A_{1}$, so $\mathcal{F}_{S} \subseteq \mathcal{F}_{T}$. Similarly, $\mathcal{F}_{T} \subseteq \mathcal{F}_{S}$. Thus we conclude that $\mathcal{F}_{S}=\mathcal{F}_{T}$.

Suppose now that $\mathcal{F}_{S}=\mathcal{F}_{T}$. Choose elements $e_{i} \in H_{i}$ such that $\left\|e_{i}\right\|=1$ and let $\mathfrak{H}$ be the subspace of $H$ generated by all $e_{i},-\infty<i<\infty$. Then $\mathfrak{H}$ is invariant for $S$ and $T, S e_{i}=s_{i} e_{i}$ and $T e_{i}=t_{i} e_{i}$. By $S_{\mathfrak{H}}$ and $T_{\mathfrak{H}}$ we denote the restrictions of $S$ and $T$ to $\mathfrak{H}$. Since $\mathcal{F}_{S}=\mathcal{F}_{T}$,

$$
\mathcal{F}_{S_{\mathfrak{H}}}=\mathcal{F}_{T_{\mathfrak{H}}}
$$

We shall show now that $M$ and $N$ are Schur multipliers on $\mathfrak{H}$.

The function $f(t)=i(\pi-t)$ on $[0,2 \pi]$ has Fourier coefficients $c_{0}=0$ and $c_{n}=\frac{1}{n}$, for $n= \pm 1, \pm 2, \ldots$. Let $\mathcal{H}$ be a Hilbert space with an orthonormal basis $\left\{h_{k}\right\}_{k=-\infty}^{\infty}$ and $R=\left(r_{k l}\right),-\infty<k, l<\infty$, be a Toeplitz matrix such that $r_{k k}=0$ and $r_{k l}=c_{k-l}=\frac{1}{k-l}, k \neq l$. Then $R \in B(\mathcal{H})$ and it follows from Theorem $8.1[\mathbf{1}]$ that $R$ is a Schur multiplier and $|R|=\sup |f(t)|=\pi$.

Identifying $e_{i}$ in $\mathfrak{H}$ with $h_{t_{i}}$ in $\mathcal{H}$, we can consider $\mathfrak{H}$ as a subspace of $\mathcal{H}$. For $B=\left(b_{k m}\right) \in B(\mathfrak{H})$, where $b_{k m}=\left(B e_{m}, e_{k}\right)$, let $\widetilde{B}=\left(\widetilde{b}_{i j}\right) \in B(\mathcal{H})$ be such that $\left.\widetilde{B}\right|_{\mathfrak{H}}=B$ and $\left.\widetilde{B}\right|_{\mathfrak{H}^{\perp}}=0$. Then $\|\widetilde{B}\|=\|B\|$,

$$
\begin{aligned}
\widetilde{b}_{t_{k} t_{m}} & =\left(\widetilde{B} h_{t_{m}}, h_{t_{k}}\right)=\left(B e_{m}, e_{k}\right)=b_{k m}, \quad \text { and } \\
\widetilde{b}_{i j} & =\left(\widetilde{B} h_{j}, h_{i}\right)=0 \text { if either } i \neq t_{k} \text { or } j \neq t_{m} .
\end{aligned}
$$

Since $R$ is a Schur multiplier, the operator $\widetilde{C}=\left(\widetilde{c}_{i j}\right)=R \circ \widetilde{B}$ belongs to $B(\mathcal{H})$, where

$$
\begin{aligned}
\widetilde{c}_{t_{k} t_{m}} & =r_{t_{k} t_{m}} \widetilde{b}_{t_{k} t_{m}}=\left(t_{k}-t_{m}\right)^{-1} b_{k m}, \quad \text { if } k \neq m, \quad \text { and } \\
\widetilde{c}_{i j} & =0 \text { if either } i \neq t_{k} \text { or } j \neq t_{m} \text { or } i=j=t_{k} .
\end{aligned}
$$

Setting $C=\left.\widetilde{C}\right|_{\mathfrak{H}}$, we obtain that $C=\left(c_{k m}\right) \in B(\mathfrak{H})$, where

$$
c_{k m}=\widetilde{c}_{t_{k} t_{m}}=\left(t_{k}-t_{m}\right)^{-1} b_{k m}, \text { if } k \neq m \text {, and } c_{k k}=0,
$$


that $\|\widetilde{C}\|=\|C\|$ and that $C=W \circ B$, where $W=\left(w_{k m}\right)$ is a matrix such that

$$
w_{k m}=\left(t_{k}-t_{m}\right)^{-1}, \quad k \neq m, \text { and } w_{k k}=0 .
$$

From this it follows that $W$ is a Schur multiplier on $\mathfrak{H}$ and

$$
\|W \circ B\|=\|C\|=\|\widetilde{C}\|=\|R \circ \widetilde{B}\| \leq|R|\|\widetilde{B}\|=|R|\|B\| .
$$

Thus $|W| \leq|R|=\pi$.

Let $P_{n}$ be the orthoprojections in $\mathfrak{H}$ on the subspaces $\sum_{j=-n}^{n} \oplus\left\{\mathbb{C} e_{j}\right\}$. Then $P_{n}$ are finite rank operators commuting with operators $S_{\mathfrak{H}}$ and $T_{\mathfrak{H}}$ and $P_{n} \mathfrak{H} \subseteq D\left(S_{\mathfrak{H}}\right)$. Hence $P_{n} \in \mathcal{F}_{S_{\mathfrak{H}}}$. For every $B \in B(\mathfrak{H}), P_{n} B P_{n}$ are finite rank operators preserving $D\left(S_{\mathfrak{H}}\right)$ and their adjoints $P_{n} B^{*} P_{n}$ also preserve $D\left(S_{\mathfrak{H}}\right)$. Therefore

$$
P_{n} B P_{n} \in \mathcal{F}_{S_{\mathfrak{H}}} .
$$

Any $B=\left(b_{k m}\right) \in B(\mathfrak{H})$ can be represented in the form $B=B_{d}+B_{0}$, where $B_{d}$ is the diagonal operator such that $\left(B_{d}\right)=b_{k k}$. Then

$$
\left\|B_{d}\right\| \leq\|B\| \quad \text { and } \quad\left\|B_{0}\right\|=\left\|B-B_{d}\right\| \leq 2\|B\| .
$$

We have that

$$
M \circ\left(P_{n} B P_{n}\right)=P_{n}(M \circ B) P_{n} .
$$

Since $m_{k k}=0$ in the matrix $M=\left(m_{k m}\right)$,

$$
M \circ\left(P_{n} B P_{n}\right)=M \circ\left(P_{n} B_{0} P_{n}\right) .
$$

Set $A=W \circ B$. Since $W$ is a Schur multiplier, $A \in B(\mathfrak{H})$ and, by (4.18), $P_{n} A P_{n} \in \mathcal{F}_{S_{\mathfrak{H}}}$. It is easy to check that

$$
\begin{aligned}
P_{n} B_{0} P_{n} & =T_{\mathfrak{H}}\left(P_{n} A P_{n}\right)-\left(P_{n} A P_{n}\right) T_{\mathfrak{H}}=\left(P_{n} A P_{n}\right)_{T_{\mathfrak{H}}}, \quad \text { and } \\
M \circ\left(P_{n} B_{0} P_{n}\right) & =S_{\mathfrak{H}}\left(P_{n} A P_{n}\right)-\left(P_{n} A P_{n}\right) S_{\mathfrak{H}}=\left(P_{n} A P_{n}\right)_{S_{\mathfrak{H}}} .
\end{aligned}
$$

Since $\mathcal{F}_{S_{\mathfrak{H}}}=\mathcal{F}_{T_{\mathfrak{H}}}$, it follows from Lemma 4.1(i) that the norms $\|\cdot\|_{S_{\mathfrak{H}}}$ and $\|\cdot\|_{T_{\mathfrak{H}}}$ are equivalent. Therefore there exists $D>0$ such that $\left\|P_{n} A P_{n}\right\|_{S_{\mathfrak{H}}} \leq D\left\|P_{n} A P_{n}\right\|_{T_{\mathfrak{H}}}$. Hence we obtain from (4.19), (4.21) and (4.22) that

$$
\begin{aligned}
\left\|M \circ\left(P_{n} B P_{n}\right)\right\| & =\left\|M \circ\left(P_{n} B_{0} P_{n}\right)\right\|=\left\|\left(P_{n} A P_{n}\right)_{S_{\mathfrak{H}}}\right\| \\
& \leq\left\|P_{n} A P_{n}\right\|_{S_{\mathfrak{H}}} \leq D\left\|P_{n} A P_{n}\right\|_{T_{\mathfrak{H}}} \\
& =D\left(\left\|P_{n} A P_{n}\right\|+\left\|\left(P_{n} A P_{n}\right)_{T_{\mathfrak{H}}}\right\|\right) \\
& \leq D\left(\|A\|+\left\|P_{n} B_{0} P_{n}\right\|\right) \leq D\left(\|A\|+\left\|B_{0}\right\|\right) \\
& =D\left(\|W \circ B\|+\left\|B_{0}\right\|\right) \leq D(\mid R\|\| B\|+2\| B \|)=\rho .
\end{aligned}
$$

Thus all operators $M \circ\left(P_{n} B P_{n}\right), 1 \leq n<\infty$, lie in the ball $\mathbf{B}_{\rho}$ of $B(\mathfrak{H})$ of radius $\rho$. Compactness of $\mathbf{B}_{\rho}$ in the weak operator topology implies that the 
sequence $\left\{M \circ\left(P_{n} B P_{n}\right)\right\}_{n=1}^{\infty}$ has a cluster point $K \in B(\mathfrak{H})$. Therefore there is a subsequence $\left\{M \circ\left(P_{n_{j}} B P_{n_{j}}\right)\right\}$ such that for all $e_{k}$ and $e_{m}$,

$$
\left(K e_{k}, e_{m}\right)=\lim _{j \rightarrow \infty}\left(M \circ\left(P_{n_{j}} B P_{n_{j}}\right) e_{k}, e_{m}\right) .
$$

If $n_{j} \geq \max (|k|,|m|)$ then $P_{n_{j}} e_{k}=e_{k}$ and $P_{n_{j}} e_{m}=e_{m}$ and, by (4.20),

$$
\left(M \circ\left(P_{n_{j}} B P_{n_{j}}\right) e_{k}, e_{m}\right)=\left(P_{n_{j}}(M \circ B) P_{n_{j}} e_{k}, e_{m}\right)=\left(M \circ B e_{k}, e_{m}\right) .
$$

Hence $\left(K e_{k}, e_{m}\right)=\left((M \circ B) e_{k}, e_{m}\right),-\infty<k, m<\infty$. Thus $K=M \circ B$, so $M$ is a Schur multiplier. Similarly, we obtain that $N$ is also a Schur multiplier.

Example 4.7. Let

$$
s_{i}=i \quad \text { and } \quad t_{i}=(-1)^{i} i
$$

in Theorem 4.6. If $\mathcal{F}_{S}=\mathcal{F}_{T}$ then, by Theorem 4.6, $M$ is a Schur multiplier and we have that $\left|m_{i j}\right| \leq|M|$ for all $i$ and $j$. Let $i=2 k$ and $j=-2 k+1$. Then $s_{i}=t_{i}=2 k$ and $s_{j}=-t_{j}=-2 k+1$. Hence

$$
m_{i j}=\frac{s_{i}-s_{j}}{t_{i}-t_{j}}=4 k-1 \rightarrow \infty, \text { as } k \rightarrow \infty .
$$

This shows that $M$ is not a Schur multiplier and, therefore, $\mathcal{F}_{S} \neq \mathcal{F}_{T}$.

Making use of Theorem 4.6, we obtain the following result of a more general character.

Theorem 4.8. Let $S$ and $T$ be selfadjoint operators on $H$ and $\mathcal{H}$ respectively. If there exists a bijection $\varphi$ of $\mathbb{Z}$ onto $\mathbb{Z}$ such that

$$
\operatorname{dim}\left(\mathcal{H}_{T}(\varphi(i))\right)=\operatorname{dim}\left(H_{S}(i)\right), \quad \text { for all } i \in \mathbb{Z},
$$

(see (2.2) for definition of $\mathcal{H}_{T}(i)$ and $\left.H_{S}(i)\right)$ and if

$$
\begin{aligned}
& M=\left(m_{i j}\right) \text { where } m_{i j}=\frac{\varphi(i)-\varphi(j)}{i-j}, \text { for } i \neq j, \text { and } m_{i j}=0, \text { and } \\
& N=\left(n_{i j}\right) \text { where } n_{i j}=\frac{i-j}{\varphi(i)-\varphi(j)}, \text { for } i \neq j, \text { and } n_{i j}=0
\end{aligned}
$$

are Schur multipliers then the algebras $\mathcal{F}_{S}$ and $\mathcal{F}_{T}$ are ${ }^{*}$-isomorphic.

Proof. Consider the operators $[S]$ and $[T]$ (see (2.1)) and the corresponding decompositions

$$
H=\sum_{i \in \mathbb{Z}} \oplus H_{S}(i) \quad \text { and } \quad \mathcal{H}=\sum_{i \in \mathbb{Z}} \oplus \mathcal{H}_{T}(i)
$$

where $H_{S}(i)=P_{S}(i) H$ and $\mathcal{H}_{T}(i)=P_{T}(i) \mathcal{H}$ (see (2.3)). The operators $S-[S]$ and $T-[T]$ are bounded, so $\mathcal{F}_{S}=\mathcal{F}_{[S]}$ and $\mathcal{F}_{T}=\mathcal{F}_{[T]}$. 
Consider the selfadjoint operator $R$ on $H$ such that all subspaces $H_{S}(i)$ are invariant for $R$ and $\left.R\right|_{H_{S}(i)}=\varphi(i) \mathbf{1}_{H_{S}(i)}$. Since $M$ and $N$ are Schur multipliers, it follows from Theorem 4.6 that $\mathcal{F}_{R}=\mathcal{F}_{[S]}$.

On the other hand, since $\operatorname{dim}\left(\mathcal{H}_{T}(\varphi(i))\right)=\operatorname{dim}\left(H_{S}(i)\right)$, for all $i \in \mathbb{Z}$, there exists an isometry operator $U$ from $H$ onto $\mathcal{H}$ which maps $H_{S}(i)$ onto $\mathcal{H}_{T}(\varphi(i))$. Then $U^{*}[T] U=R$. By Lemma 4.1, the algebras $\mathcal{F}_{R}$ and $\mathcal{F}_{[T]}$ are *-isomorphic. Hence the algebras $\mathcal{F}_{S}$ and $\mathcal{F}_{T}$ are ${ }^{*}$-isomorphic.

\section{References}

[1] G. Bennett, Schur multipliers, Duke Math. J., 44 (1977), 603-639.

[2] B. Blackadar and J. Cuntz, Differential Banach algebra norms and smooth subalgebras of $C^{*}$-algebras, J. Oper. Theory, 26 (1991), 255-282.

[3] O. Bratteli and D.W. Robinson, Unbounded derivations of $C^{*}$-algebras, I, Comm. Math. Phys., 42 (1975), 253-268.

[4] J. Dixmier, Les $C^{*}$-algebres et leurs Representations, Gauthier-Villars, Paris, 1969.

[5] N. Dunford and J.T. Schwartz, Linear Operators, Vol. I+II, Interscience Inc., New York, 1988.

[6] I.Ts. Gohberg and M.G. Krein, Introduction to the theory of linear non-selfadjoint operators in Hilbert spaces, Nauka, Moscow, 1965.

[7] P.E.T. Jorgensen and G.L. Price, Index theory and second quantization of boundary value problems, J. Funct. Anal., 104 (1992), 243-290.

[8] E. Kissin, Symmetric operator extensions of unbounded derivations of $C^{*}$-algebras, J. Funct. Anal., 81 (1988), 38-53.

[9] _ Indices of unbounded derivations of $C^{*}$-algebras, Pacific J. Math., 152 (1992), 125-150.

[10] E. Kissin, A.I. Loginov and V.S. Shulman, Derivations of $C^{*}$-algebras and almost Hermitian representations on $\Pi_{k}$-spaces, Pacific J. Math., 174 (1996), 411-430.

[11] E. Kissin and V.S. Shulman, Dense Q-subalgebras of Banach and $C^{*}$-algebras and unbounded derivations of Banach and $C^{*}$-algebras, Proc. Edinburgh Math. Soc., 36 (1993), 261-276.

[12] _ Differential properties of some dense subalgebras of $C^{*}$-algebras, Proc. Edinburgh Math. Soc., 37 (1994), 399-422.

[13] Differential Banach *-algebras of compact operators associated with symmetric operators, J. Funct. Anal., 156 (1998), 1-29.

[14] Representations on Krein Spaces and Derivations of $C^{*}$-algebras, Addison Wesley Longman, London, 1997.

[15] M. Reed and B. Simon, Methods of Modern Mathematical Physics, Academic Press, New York London, 1972. 
[16] S. Sakai, Operator Algebras in Dynamical Systems, CUP, Cambridge, 1991.

Received December 10, 1997 and revised September 16, 1998.

UNIVERSITY OF NORTH LONDON

Great Britain

E-mail address: 11kissine@unl.ac.uk

Polytechnic Institute of Vologda

VOLOGDA

Russia 